hep-th/9908046

August 1999

\title{
Structure constants for the D-series Virasoro minimal models
}

\author{
Ingo Runkel[ \\ Mathematics Department, \\ King's College London, Strand, London WC2R 2LS, U.K.
}

\begin{abstract}
In this paper expressions are given for the bulk and boundary structure constants of Dseries Virasoro minimal models on the upper half plane. It is the continuation of an earlier work on the A-series. The solution for the boundary theory is found first and then extended to the bulk. The modular invariant bulk field content is recovered as the maximal set of bulk fields consistent with the boundary theory. It is found that the structure constants are unique up to redefinition of the fields and in the chosen normalisation exhibit a manifest $\mathbb{Z}_{2}$-symmetry associated to the D-diagram. The solution has been subjected to random numerical tests against the constraints it has to fulfill.
\end{abstract}

${ }^{1}$ e-mail: ingo@mth.kcl.ac.uk 


\section{Introduction}

One of the aims of studying quantum field theories is to compute correlation functions of the fields in the theory. In two-dimensional conformal field theory one uses the presence of an infinite amount of symmetries to achieve this goal.

We will consider a special subclass of conformal field theories, called minimal models. One of their properties is that the correlators fulfill certain linear differential equations (see [1] or e.g. [2] for details). One can find a distinguished set of solutions to these differential equations, called conformal blocks. A correlator can then be expressed as a bilinear combination of these blocks. To know the coefficients in this bilinear combination a set of complex numbers, the structure constants, are needed. Structure constants appear in the short distance expansion (or operator product expansion, OPE) of certain fields in the conformal field theory, called primary fields.

In this paper we consider only Virasoro minimal models. One approach to calculate all structure constants is to start in a situation without boundaries. In this case only bulk fields are present and one can solve the bulk theory alone by determining the conformal blocks and a consistent set of structure constants for the bulk fields (see [3] for the spinless case). It is possible to classify all modular invariant minimal models without boundaries [4] with the result that they fall into an $\mathrm{A}-\mathrm{D}-\mathrm{E}$ pattern.

Starting from a well defined bulk theory one can proceed and introduce a boundary into the system. As it turns out the differential equations that the correlators fulfill stay the same, but now the correlator is a linear, not a bilinear, combination of the conformal blocks mentioned above (see [5]) and furthermore two new sets of structure constants appear: In addition to the bulk structure constants that determine the OPE of two bulk primary fields, there are the bulk-boundary couplings that describe the expansion of a bulk field in terms of boundary fields, and the boundary structure constants for the OPE of two boundary fields.

One can now classify all conformally invariant boundary conditions and the boundary fields that live on these boundaries or interpolate between two adjacent boundary conditions (see [6 9]). Again one finds an A-D-E pattern for all possible boundary theories [9], plus tadpole diagrams, which have been discarded as the resulting theory does not match with any of the modular invariant bulk theories.

All three sets of structure constants can in principle be worked out from six consistency conditions that arise from taking different limits in certain correlators and equating the different OPEs in these limits. The constraints that arise in this way are called sewing constraints (see [10] and for an extension to non-orientable surfaces [7]). A more general discussion of the sewing constraints in presence of nontrivial multiplicities and their relation to [11] can be found in [12].

Above we have started from a bulk theory, which can be solved consistently, and then introduced a boundary. Thus the bulk structure constants are known first and then a solution for the other two sets of structure constants consistent with these has to be obtained. Whereas the bulk structure constants for diagonal and nondiagonal minimal models can be found in the literature [3, 13, 14], these calculations have not been carried out for the boundary structure constants and only for a subset of the bulk-boundary couplings (see [6 9]).

In this paper and a previous work [15] the missing structure constants for the A- and Dseries are calculated by taking the opposite path. The starting point now is a theory with only boundary fields. One chooses an A-D-E type boundary field content and tries to determine a consistent set of boundary structure constants. Once this is achieved the boundary theory is extended to the bulk and it is only at this point that bulk fields are introduced. It is 
interesting that one finds that the maximal bulk field content consistent with the boundary theory coincides with the modular invariant one given in 4 . The bulk-boundary couplings and the bulk structure constants can now be obtained from the sewing constraints in a straight forward way. The results are explicit expressions for all three sets of structure constants in terms of so called fusion ( $\left.\mathrm{F}_{-}\right)$matrices (see Appendix A for details and references). The expressions are free of sign ambiguities (e.g. they do not contain any square roots) so that they are well suited for numerical computations.

The $D_{\text {even }}$ minimal models have a larger symmetry algebra (a W-algebra, see [16] and references therein) that contains the Virasoro algebra as subalgebra. In principle one could understand these conformal field theories in terms of the $\mathrm{W}$-algebra, with $\mathrm{W}$-algebra chiral blocks and $\mathrm{F}$-matrices. But since these CFTs are still finitely reducible with respect to the Virasoro algebra alone (i.e. only a finite number of irreducible highest weight representations of the Virasoro algebra occur), it is possible and indeed simple to use only the Virasoro symmetry.

The fact that one does not use the fully extended chiral algebra and the possible presence of multiple copies of primary fields means that the sewing constraints in Ref. [10] must be rederived, which has been done. The results are presented in section 2 and an explicit example is provided in Appendix A.

Both, in the $\mathrm{A}$ - and the $\mathrm{D}$-series, the sewing constraints are overdetermined and only a subset is used to find the structure constants. It has to some extend been tested numerically (but not proven analytically) that the given structure constants solve the full set of constraints. It is shown that, given the boundary field content, any solution can be brought to a standard form by redefining the fields. In other words, under gauge transformations the space of solutions to the sewing constraints consists of only one orbit.

The formulae for all bulk and boundary structure constants are given in eqns. (43)-(47) and (53)-(55). A short description of how to implement them can be found in the conclusion. The derivation of these expressions is organised as follows:

In section 2 the notation for the various fields and structure constants is introduced and the rederived sewing constraints for genus zero surfaces are stated in a suitable form. Section 3 gives a short description of the procedure introduced in [9] for the construction of cylinder partition functions. This defines the boundary field content which is used as input in the construction of the solution to the sewing constraints. The boundary structure constants are found in section 1 . Finally the extension to the bulk theory is carried out in section 5. Here the maximal bulk field content consistent with the boundary theory is determined and it is observed to be modular invariant. The remaining structure constants, i.e. the bulk-boundary couplings and the bulk structure constants are calculated and expressions for the vacuum expectation value of the unit disc are obtained. A choice of basis is presented which renders

all structure constants real. Finally it is observed that a subset of all structure constants is left invariant under a large number of distinct $\mathbb{Z}_{2}-$ actions.

\section{Genus zero sewing constraints}

Unless otherwise mentioned, throughout this paper the boundary conformal field theory will be considered on the upper half plane (UHP) with the boundary given by the real line.

In a general Virasoro minimal model there can be several primary bulk or boundary fields transforming in the same highest weight representation(s) of the Virasoro algebra. Therefore we cannot label the fields solely by their representations. Virasoro highest weight representations are labelled by small letters $i, j, k$ and to take care of fields with multiplicities these 
carry an additional Greek index $i_{\alpha}, j_{\beta}, k_{\gamma}$. Boundary conditions are labelled by small letters $a, b, c$ or also $x, y, z$.

It is always possible to make the (bulk- and boundary-) two-point functions diagonal by an appropriate choice of labels and normalisation of the fields: $\left\langle\varphi_{i_{\alpha}}(x) \varphi_{j_{\beta}}(y)\right\rangle=\delta_{i, j} \delta_{\alpha, \beta} \cdot f(x, y)$.

A bulk field $\phi_{i_{\alpha}}$ transforms in the tensor product of two Virasoro highest weight representations $i \otimes \bar{\imath}$. The two representations have conformal weights $h_{i}, h_{\bar{\imath}}$ or, for better readability, $h_{i}, \bar{h}_{i}$. A boundary field $\psi_{k_{\alpha}}^{(a b)}$ lives between boundary conditions $a$ and $b$ and transforms in one Virasoro representation $k$ of conformal weight $h_{k}$.

The vacuum expectation value of the UHP with boundary condition $a$ imposed on the real line will be denoted with $\langle 1\rangle_{\mathrm{UHP}}^{(a)}$. The vacuum expectation value of the full complex plane with no boundaries present will be denoted with $\langle 1\rangle$ or $\langle 0 \mid 0\rangle$.

The leading terms in the bulk-bulk, bulk-boundary and boundary-boundary operator product expansions of primary fields are, in this order:

$$
\begin{aligned}
\phi_{i_{\alpha}}(z) \phi_{j_{\beta}}(w) & =\sum_{k, \gamma} C_{i_{\alpha} j_{\beta}}{ }^{k_{\gamma}}(z-w)^{h_{k}-h_{i}-h_{j}}(\bar{z}-\bar{w})^{\bar{h}_{k}-\bar{h}_{i}-\bar{h}_{j}} & & \left(\phi_{k_{\gamma}}(w)+\cdots\right) \\
\phi_{i_{\alpha}}(x+i y) & =\sum_{k, \gamma}{ }^{(a)} B_{i_{\alpha}}{ }^{k}{ }^{k_{\gamma}} \cdot(2 y)^{h_{k}-h_{i}-\bar{h}_{i}} \cdot\left(\psi_{k_{\gamma}}^{(a a)}(x)+\cdots\right) & & |z|>|w| \\
\psi_{i_{\alpha}}^{(a b)}(x) \psi_{j_{\beta}}^{(b c)}(y) & =\sum_{k, \gamma} C_{i_{\alpha} j_{\beta}}^{(a b c) k_{\gamma}} \cdot(x-y)^{h_{k}-h_{i}-h_{j}} \cdot\left(\psi_{k_{\gamma}}^{(a c)}(y)+\cdots\right) & & x>y
\end{aligned}
$$

The omissions stand for an infinite sum of descendants of the primary field in question. Eqn. (11)-(3) define the three sets of structure constants which are necessary to compute the correlation functions of the minimal model under consideration: the bulk structure constants $C_{i_{\alpha} j_{\beta}}{ }^{k_{\gamma}}$ with three bulk fields $i_{\alpha}, j_{\beta}, k_{\gamma}$, the bulk-boundary couplings ${ }^{(a)} B_{i_{\alpha}}{ }^{{ }}$with boundary condition $a$, bulk field $i_{\alpha}$ and boundary field $k_{\gamma}$ and the boundary structure constants $C_{i_{\alpha} j_{\beta}}^{(a b c) k_{\gamma}}$ with boundary conditions $a, b, c$ and boundary fields $i_{\alpha}, j_{\beta}, k_{\gamma}$.

By taking different limits in correlation functions one obtains a set of constraints, called sewing constraints. For orientable genus zero surfaces it is enough to consider the following four constraints [10]:

For four boundary fields $i_{\alpha}, j_{\beta}, k_{\gamma}, \ell_{\delta}$ and boundary conditions $a, b, c, d$ :

$$
\begin{aligned}
\sum_{\epsilon} C_{j_{\beta} k_{\gamma}}^{(b c d) q_{\epsilon}} C_{i_{\alpha} q_{\epsilon}}^{(a b d) \ell_{\delta}} C_{\ell_{\delta} \ell_{\delta}}^{(a d d a) 1}\langle 1\rangle_{\mathrm{UHP}}^{(a)} & \\
= & \sum_{p}\left(\sum_{\nu} C_{i_{\alpha} j_{\beta}}^{\left(a b c p_{\nu}\right.} C_{k_{\gamma} \ell_{\delta}}^{(c d a) p_{\nu}} C_{p_{\nu} p_{\nu}}^{(a c a) 1}\langle 1\rangle_{\mathrm{UHP}}^{(a)}\right) \mathrm{F}_{p q}\left[\begin{array}{cc}
j & k \\
i & \ell
\end{array}\right]
\end{aligned}
$$

For two boundary fields $p_{\nu}, q_{\epsilon}$ and one bulk field $i_{\alpha}$ :

$$
\begin{aligned}
\sum_{\delta}{ }^{(b)} B_{i_{\alpha}}{ }^{\ell_{\delta}} & C_{p_{\nu} \ell_{\delta}}^{(a b b) q_{\epsilon}} C_{q_{\epsilon} q_{\epsilon}}^{(a b a) 1}\langle 1\rangle_{\mathrm{UHP}}^{(a)} \\
=\sum_{k} & \left(\sum_{\gamma}{ }^{(a)} B_{i_{\alpha}}{ }^{k_{\gamma}} C_{p_{\nu} q_{\epsilon}}^{(a b a) k_{\gamma}} C_{k_{\gamma} k_{\gamma}}^{(a a a) 1}\langle 1\rangle_{\mathrm{UHP}}^{(a)}\right) \\
& \cdot \sum_{m} e^{i \pi\left(2 h_{m}-2 h_{i}-h_{p}-h_{q}+\frac{1}{2}\left(h_{k}+h_{\ell}\right)\right)} \cdot \mathrm{F}_{k m}\left[\begin{array}{cc}
\bar{\imath} & q \\
i & p
\end{array}\right] \mathrm{F}_{m \ell}\left[\begin{array}{cc}
i & \bar{\imath} \\
p & q
\end{array}\right]
\end{aligned}
$$


For two bulk fields $i_{\alpha}, j_{\beta}$ and one boundary field $k_{\gamma}$ :

$$
\begin{aligned}
& \sum_{\rho} C_{i_{\alpha} j_{\beta}}{ }^{m_{\rho}}{ }^{(a)} B_{m_{\rho}}{ }^{k_{\gamma}} C_{k_{\gamma} k_{\gamma}}^{(a a a) 1}\langle 1\rangle_{\mathrm{UHP}}^{(a)} \\
& =\sum_{p, q}\left(\sum_{\nu, \epsilon}{ }^{(a)} B_{i_{\alpha}}{ }^{p_{\nu}}{ }^{(a)} B_{j_{\beta}}{ }^{q_{\epsilon}} C_{p_{\nu} q_{\epsilon}}^{(a a a) k_{\gamma}} C_{k_{\gamma} k_{\gamma}}^{(a a a) 1}\langle 1\rangle_{\mathrm{UHP}}^{(a)}\right) \\
& \cdot \sum_{r} e^{i \frac{\pi}{2}\left(h_{k}+h_{p}-h_{q}-2 h_{r}+h_{m}-\bar{h}_{m}-h_{i}+\bar{h}_{i}+h_{j}+\bar{h}_{j}\right)} \\
& \cdot \mathrm{F}_{q r}\left[\begin{array}{ll}
k & \bar{\jmath} \\
p & j
\end{array}\right] \mathrm{F}_{p m}\left[\begin{array}{ll}
\bar{\imath} & r \\
i & j
\end{array}\right] \mathrm{F}_{r \bar{m}}\left[\begin{array}{ll}
\bar{\imath} & \bar{\jmath} \\
m & k
\end{array}\right]
\end{aligned}
$$

For four bulk fields $i_{\alpha}, j_{\beta}, k_{\gamma}, \ell_{\delta}$ :

$$
\begin{aligned}
\sum_{\epsilon} C_{i_{\alpha} k_{\gamma}}{ }^{q_{\epsilon}} C_{j_{\beta} \ell_{\delta}}{ }^{q_{\epsilon}} C_{q_{\epsilon} q_{\epsilon}}{ }^{1}\langle 1\rangle & \\
=\sum_{p, \bar{p}} & \left(\sum_{\nu} C_{i_{\alpha} j_{\beta}}{ }^{p_{\nu}} C_{k_{\gamma} \ell_{\delta}}^{p_{\nu}} C_{p_{\nu} p_{\nu}}{ }^{1}\langle 1\rangle\right) \\
\cdot & e^{i \pi\left(h_{i}-\bar{h}_{i}+h_{\ell}-\bar{h}_{\ell}-h_{p}+\bar{h}_{p}-h_{q}+\bar{h}_{q}\right)} \mathrm{F}_{p q}\left[\begin{array}{cc}
j & \ell \\
i & k
\end{array}\right] \mathrm{F}_{\bar{p} \bar{q}}\left[\begin{array}{cc}
\bar{\jmath} & \bar{\ell} \\
\bar{\imath} & \bar{k}
\end{array}\right]
\end{aligned}
$$

These constraints have been rederived using notation and techniques for calculating with conformal blocks presented in [11]. Equations (4)-(7) require a specific renormalisation of the $\mathrm{F}$-matrices. This normalisation, together with the explicit derivation of the constraint (6) as an example are given in Appendix A.

The form of the constraints given here is slightly different from [10. First of all only the Virasoro symmetry was used in their derivation and not the maximally extended chiral algebra. Secondly slightly different limits in the correlators were chosen so that that the expectation value of the identity field cancels from all expressions. Furthermore the present notation takes into account fields with multiplicities (see also [12]). Leaving out the sums over multiplicities and the multiplicity indices, (伍)-(可) can be rearranged to match the corresponding equations in [10].

\section{Cylinder partition function}

First a note about a convention used throughout this paper. To take care of the redundancy in the labelling of representations $i$ with Kac-labels $(r, s)$ we will only consider pairs where $r$ is odd. The set of entries in the Kac-table with $r$ odd is in one-to-one correspondence to Virasoro highest weight representations of the given central charge.

Before we can begin to solve (44)-(团) we need to know which fields are present in the theory. Since our starting point is going to be the boundary theory the first thing to determine is the boundary field content on the upper half plane or, equivalently, the cylinder partition function.

In Ref. [9] a method for the construction of the cylinder partition functions associated to a pair of Lie-algebras $A_{n}, G$ is given. Here we give a quick summary of this method. 


\subsection{General construction}

First choose an odd number $p$ and a number $q$ coprime to $p$. We will construct the cylinder partition function of a minimal model with the following central charge:

$$
c=1-6 \frac{(p-q)^{2}}{p q}
$$

Let $A$ be the adjacency matrix of the Dynkin diagram associated to the Lie-algebra $A_{p-1}$ and $G$ be the adjacency matrix for a Lie-algebra with Coxeter number $q$.

For $X=A$ or $X=G$ define the matrix valued functions $V_{n}(X)$ recursively via

$$
V_{n}(X)=V_{2}(X) V_{n-1}(X)-V_{n-2}(X) \quad ; V_{1}(X)=\text { id and } V_{2}(X)=X
$$

The $V_{n}$ are called fused adjacency matrices and form a representation of the Verlinde fusion algebra.

Let $\alpha$ be an odd node of $A$ and $\beta$ be any node of $G$. Then $a=(\alpha, \beta)$ labels the possible boundary conditions. Let $a=(\alpha, \beta)$ and $b=(\bar{\alpha}, \bar{\beta})$. Then the partition function of a cylinder of circumference $T$ and length $L$ with boundary conditions $a$ and $b$ is given by:

$$
Z_{a \mid b}=\sum_{\substack{r=1 . . p-1 \\ s=1 . . q-1}} V_{r}(A)_{\alpha}{ }^{\bar{\alpha}} V_{s}(G)_{\beta}{ }^{\bar{\beta}} \cdot \chi_{r, s}(q) \quad ; q=\exp (-\pi T / L)
$$

When we take the identity $\chi_{r, s}=\chi_{p-r, q-s}$ for Virasoro characters into account we can rewrite (10) in the following unique way:

$$
Z_{a \mid b}=\sum_{i=(r \text { odd }, s)} n_{i a}{ }^{b} \cdot \chi_{i}(q)
$$

In Refs. [9] the genus one sewing constraint for a cylinder with no field insertions was analysed with the result that $G$ has to be the adjacency matrix of an A-D-E type Dynkin diagram or of a tadpole diagram.

The numbers $n_{i a}{ }^{b}$ can be interpreted as the number of times the representation $i$ occurs in between the boundary conditions $a$ and $b$ and they thus describe the field content on the boundary (in this case the real line).

\subsection{Example: the A-series}

For the pair of Lie algebras $\left(A_{p-1}, A_{q-1}\right)$ one finds $n_{i a}{ }^{b}=\mathrm{N}_{i a}{ }^{b}$, i.e. the field content is just given by the Verlinde fusion numbers [6]. Distinct boundary conditions are given by pairings of an odd node in the first A-diagram with any node in the second:

$$
\left\{\odot^{1} \bullet^{2} \odot \cdots \odot \bullet^{p-1}, \odot^{1} \odot^{2} \odot \cdots \odot \odot^{q-1}\right\}
$$

There is a distinguished boundary condition, which we will call 1-boundary, that corresponds to the first node in each $A$-diagram, i.e. the $(1,1)$-node.

There are no fields with multiplicity and there is a unique field $i=a$ between the $a$ - and 1-boundary: $a^{i} 1 \quad \Rightarrow i=a$. The formula $n_{i a}{ }^{b}=\mathrm{N}_{i a}{ }^{b}$ can be understood as saying that the representations $i$ that can live between the $a$ - and $b$-boundary are exactly those occurring

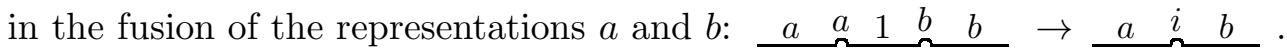




\subsection{The D-series}

Here $q$ has to be an even number and the boundary conditions are given by pairings of an odd node in the A-diagram with any node in the D-diagram:

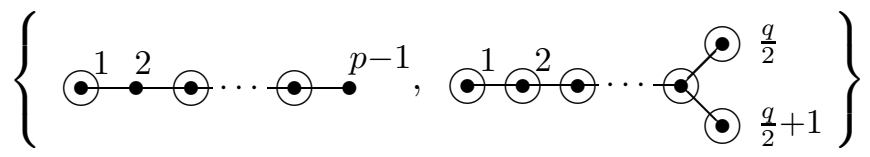

In particular the total number of boundary conditions is $\frac{1}{4}(p-1)(q+2)$. Again the boundary condition associated with the first node of each diagram, i.e. the $(1,1)$-node, will get a special name. It will be called $\omega$ so that it is not confused with the 1-boundary in the A-series. The $\omega$-boundary will play a role similar to that of the 1-boundary in [15].

When looking at the D-series boundary field content one observes the following: The boundary conditions can be organised in two categories. In the first case, which we will denote as 'i-type' boundaries, a boundary condition $x=(\alpha, \beta)$ is associated with an odd node $\alpha$ of the $A$-diagram and any node $\beta$ of the $D$-diagram except for the two at the split end. The second case we will call 'n-type'. An n-type boundary $a$ is associated with any odd $A$-node and one of the two end nodes in the $D$-diagram.
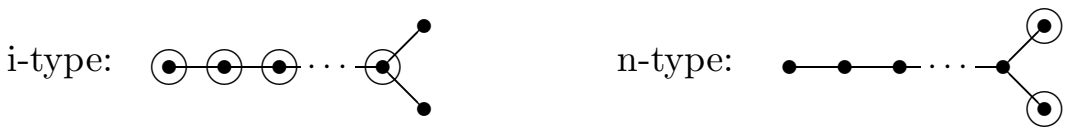

The names i-type and n-type stand for 'invariant' and 'non-invariant' and are related to the $\mathbb{Z}_{2}$-symmetry of the D-diagram.

Carrying through the procedure outlined in section 3.1 one finds that an n-type boundary $a=(\alpha, \beta)$ has exactly one field living on $\quad a \_\omega$, denoted by $a_{u}$ ('unique'). It has representation labels $\left(\alpha, \frac{q}{2}\right)$.

An i-type boundary $x=(\alpha, \beta)$ has two fields fields living on $x \sim \omega$ with Kac-labels $(\alpha, \beta)$ and $(\alpha, q-\beta)$ which we will denote by $x_{e}$ and $x_{o}$ ( $x$ 'even' and $x$ 'odd'). The labelling is arbitrary, but we choose to always give the identity field on the $\omega$-boundary an 'even' label.

Note that since the Kac-labels of the representations that live on $\quad x \quad \omega$ terms of the node labels of the boundary condition $x=(\alpha, \beta)$ some confusion might arise. But from the context it will be clear which one of the two interpretations makes sense.

For definiteness we will fix a specific even/odd labelling for boundary fields. It will turn out later that the structure constants involving only i-type boundaries have an explicit $\mathbb{Z}_{2}-$ symmetry that sends even fields to themselves and odd fields to minus themselves. This symmetry is not assumed, but a consequence of the constraints and the gauge we choose. Following the arguments in 19] we would like the ground state, i.e. the state of lowest conformal weight, for each pair of boundary conditions to be invariant under that symmetry. This can be achieved for all pairs $\quad x \quad \omega$ with $x=(\alpha, \beta)$ an i-type boundary by assigning $e / o$-labels to the two representations living between these boundary conditions in the following way:

$$
x_{e}=\left\{\begin{array}{ll}
(\alpha, \beta) & : \alpha<\frac{p}{2} \\
(\alpha, q-\beta) & : \alpha>\frac{p}{2}
\end{array} \quad x_{o}= \begin{cases}(\alpha, q-\beta) & : \alpha<\frac{p}{2} \\
(\alpha, \beta) & : \alpha>\frac{p}{2}\end{cases}\right.
$$

As discussed in [19] the physical motivation for this choice comes from relating the $\mathbb{Z}_{2}$-action to the effect of a disorder line stretching from boundary to boundary on a cylinder and trying to interpret the resulting amplitude as a partition function. It is also found there that in 


\begin{tabular}{|c|c|c|c|}
\hline bou & dary types & $\ell$-labels & field content \\
\hline $\mathrm{i}-\mathrm{i}$ & $\begin{array}{lll}x & \ell & y \\
\end{array}$ & $\mathrm{e}, \mathrm{O}$ & $n_{\ell x^{y}}=\mathrm{N}_{\ell x_{e}}{ }^{y_{e}}+\mathrm{N}_{\ell x_{e}}{ }^{y_{o}}=\mathrm{N}_{\ell x_{o}}{ }^{y_{o}}+\mathrm{N}_{\ell x_{o}}{ }^{y_{e}}$ \\
\hline$n-i$ & $a \quad \ell \quad y$ & $\mathrm{u}$ & $n_{\ell a^{y}}^{y}=\mathrm{N}_{\ell a_{u}}^{y_{e}}=\mathrm{N}_{\ell a_{u}}^{y_{o}}$ \\
\hline$n-n$ & $a \quad \ell \quad b$ & $\mathrm{u}$ & $\begin{aligned} \beta_{a} \neq \beta_{b}: & n_{\ell a}{ }^{b}=\mathrm{N}_{\ell a_{u}} b_{u} \text { if } s_{\ell} \equiv 3 \bmod 4 \\
& \text { and } n_{\ell a}{ }^{b}=0 \text { otherwise } \\
\beta_{a}=\beta_{b}: & n_{\ell a}{ }^{a}=\mathrm{N}_{\ell a_{u}}{ }^{a_{u}} \text { if } s_{\ell} \equiv 1 \bmod 4 \\
& \text { and } n_{\ell a}{ }^{b}=0 \text { otherwise }\end{aligned}$ \\
\hline
\end{tabular}

Table 1: Field content and labels between the two types of boundary conditions.

general it is not possible to fix a labelling s.t. the ground state is invariant for all possible pairs of boundary conditions.

The conformal weight of a highest weight representation with Kac-labels $(r, s)$ is given by $h_{r, s}=\frac{1}{4 p q}\left((q r-p s)^{2}-(p-q)^{2}\right)$. Thus with definition (15) we find:

$$
h\left(x_{o}\right)-h\left(x_{e}\right)=\left|\left(\alpha-\frac{p}{2}\right)\left(\beta-\frac{q}{2}\right)\right|
$$

In particular the $\omega$-boundary itself is of i-type and it has two fields living on it, the identity $\omega_{e}=1$ and the field $\omega_{o}$ with $h\left(\omega_{o}\right)=\left(\frac{p}{2}-1\right)\left(\frac{q}{2}-1\right)$. We see that $h\left(\omega_{o}\right)$ is integer for $\frac{q}{2}$ odd and half-integer for $\frac{q}{2}$ even.

We will now proceed to assign e/o/u-labels to all boundary fields, not just the ones adjacent to the $\omega$-boundary, in the following way: Any field adjacent to an n-type boundary will get the label $u$. In this case no multiplicities do occur. In the case $\underbrace{x \ell_{\delta} y}$ where both $x$ and $y$ are of i-type, the possible representations $\ell$ are those that occur in the fusion of $x_{e}, y_{e}$ (which is the same set as in the fusion of $x_{o}, y_{o}$ ) or $x_{e}, y_{o}$ (which is the same as $x_{o}, y_{e}$ ). If $\ell$ occurs in the $x_{e}, y_{e}$-fusion it gets an $e$-label, i.e. $\delta=e$ and if it occurs in the $x_{e}, y_{o}$-fusion an $o$-label $\delta=o$. If $\ell$ occurs in both fusions, this representation has multiplicity two and the two corresponding fields have labels $\ell_{e}$ and $\ell_{o}$.

The field content between different boundary conditions is summed up in table 1 listing the numbers $n_{i a}{ }^{b}$ from (11) for the different cases (here the representation $\ell$ has Kac-labels $\ell=\left(r_{\ell}, s_{\ell}\right)$ and the boundary conditions $a, b$ have labels $\left(\alpha_{a}, \beta_{a}\right)$ and $\left.\left(\alpha_{b}, \beta_{b}\right)\right)$.

Notice the double role of the $e / o / u$-indices: When $x$ is a boundary condition, then $x_{e}, x_{o}$ or $x_{u}$ denote the even/odd/unique field living between the $x$ - and $\omega$-boundary. In particular $x_{e}$ and $x_{o}$ denote different representations. If $\ell$ is a representation, then $\ell_{e}, \ell_{o}$ or $\ell_{u}$ all denote the same representation and distinguish fields with multiplicities by labelling one as even and the other as odd.

\subsection{Ordering of boundary conditions}

The constraint equations (4)-(7) allow for a large amount of gauge freedom of the structure constants. Here we find a particular set of structure constants and show that any solution of the constraints can be transformed into this set by a regauging. For the construction of the structure constants presented here an ordering of the boundary conditions has to be introduced. Let $x=(\alpha, \beta)$ and $y=(\bar{\alpha}, \bar{\beta})$. Then we define:

$$
x<y \quad \Leftrightarrow \quad(\beta<\bar{\beta}) \text { or }(\beta=\bar{\beta} \text { and } \alpha<\bar{\alpha})
$$




\section{Boundary structure constants}

In this section we will construct a set of boundary structure constants that solve the sewing constraint (4) under the condition that there exists a solution at all (for the given field content). The line of argument in this section is as follows: We assume that there exists a solution to (四) for the D-series field content. Then we use the property of (4) that a change of basis in the set of primary boundary fields maps a solution of (4) to a new solution. By redefining the boundary fields one by one this freedom is exploited to adjust a subset of boundary structure constants to the values we want. This subset together with (41) then fixes the value of a general boundary structure constant. Altogether the statement is that any solution can via change of basis be brought to the form presented in the end of this section.

As input for the boundary solution we use

a) the $D$-series field content obtained in section 3.3

b) the assumption that all two-point functions $\left\langle\psi_{i_{\alpha}}^{(a b)}(x) \psi_{i_{\alpha}}^{(b a)}(y)\right\rangle$ are nonzero, i.e. $C_{i_{\alpha} i_{\alpha}}^{(a b b) 1} \neq 0$ for all $a, b, i_{\alpha}$ that are allowed by a).

An interpretation of assumption b) is that a zero two-point function implies that the field in question does not occur in our model and hence we are looking at a field content different from what we demanded in a). This can be seen as follows: Suppose $C_{i_{\alpha} i_{\alpha}}^{(a b a) 1}=0$ for some choice of $a, b, i_{\alpha}$. Consider a correlator which contains the field $\psi_{i_{\alpha}}^{(a b)}$. Any correlator can be expressed as a sum of conformal blocks with coefficients given as products of structure constants. It is always possible to take a limit of this correlator where all bulk fields are taken to the boundary and then all boundary fields except for $\psi_{i_{\alpha}}^{(a b)}$ are taken together. In the end we are left with the two-point function $\left\langle\psi_{i_{\alpha}}^{(a b)}(x) \psi_{i_{\alpha}}^{(b a)}(y)\right\rangle$. Thus in this limit the coefficient in front of each conformal block contains the factor $C_{i_{\alpha} i_{\alpha}}^{(a b a)}$ and hence the correlator is identically zero. Therefore any correlator involving $\psi_{i_{\alpha}}^{(a b)}$ vanishes and this field can as well be removed from the model, so that we effectively have a field content different from a).

The actual calculation of the boundary structure constants is a slightly lengthy case by case study and is presented only for completeness. The results are summerised in section 4.5.

\subsection{Nonzero boundary structure constants}

First we investigate the consequence of b), i.e. that all two-point functions are nonzero. Eqn. (4) can be rewritten so that the two-point structure constants cancel. To do so we set $\ell=1$ and obtain the three-point identity:

$$
\underbrace{a i_{\alpha} \quad b \quad j_{\beta} \quad c k_{\gamma} \quad a} \Rightarrow C_{j_{\beta} k_{\gamma}}^{(b c a) i_{\alpha}} C_{i_{\alpha} i_{\alpha}}^{(a b a) 1}=C_{i_{\alpha} j_{\beta}}^{\left(a b c k_{\gamma}\right.} C_{k_{\gamma} k_{\gamma}}^{(a c a) 1}
$$

Applying this to the r.h.s. of (14) we get:

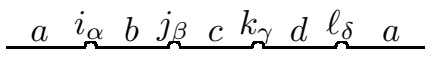

$$
\begin{aligned}
& \Rightarrow \quad \sum_{\epsilon} C_{j_{\beta} k_{\gamma}}^{(b c d) q_{\epsilon}} C_{i_{\alpha} q_{\epsilon}}^{(a b d) \ell_{\delta}}=\sum_{p, \nu} C_{i_{\alpha} j_{\beta}}^{(a b c) p_{\nu}} C_{p_{\nu} k_{\gamma}}^{(a c d) \ell_{\delta}} \mathrm{F}_{p q}\left[\begin{array}{cc}
j & k \\
i & \ell
\end{array}\right]
\end{aligned}
$$

Note that there is no freedom the rescale the identity field 1 on any boundary. Its normalisation is already fixed by the condition that $1 \cdot \psi_{i_{\alpha}}^{(x y)}=\psi_{i_{\alpha}}^{(x y)}$, i.e. that $C_{1 i_{\alpha}}^{(x x y) j_{\beta}}=\delta_{i, j} \delta_{\alpha, \beta}$ and similar for $C_{i_{\alpha} 1}^{(x y y) j_{\beta}}$. 
Define an operation '*', on the labels $e, o, u$ as follows: $x_{e}{ }^{*}=x_{o}, x_{o}{ }^{*}=x_{e}$ and $x_{u}{ }^{*}=x_{u}$, i.e. in terms of Kac-labels: $(r, s)^{*}=(r, q-s)$. Take $\underbrace{\omega \omega_{o} \omega x_{i} x x_{i} \omega \omega_{o} \omega}$ with $q=1$ and $x$ any boundary condition. The sum on the r.h.s. of (19) reduces to $p_{\nu}=x_{i}{ }^{*}$ because the representations $x_{i}$ and $\omega_{o}$ can fuse only to $x_{i}{ }^{*}$. We get:

$$
C_{x_{i} x_{i}}^{(\omega x \omega) 1}=C_{\omega_{o} x_{i}}^{(\omega \omega x) x_{i}{ }^{*}} C_{x_{i}{ }^{*} x_{i}}^{(\omega x \omega) \omega_{o}} F_{x_{i}{ }^{*} 1}\left[\begin{array}{cc}
\omega_{o} & \omega_{o} \\
x_{i} & x_{i}
\end{array}\right] \neq 0
$$

The l.h.s. is nonzero due to assumption b) and hence all terms on the r.h.s. have to be nonzero for a solution with properties a) and b).

In the same way, for an n-type boundary $a$ and an arbitrary boundary $x$ we can consider $\underbrace{\omega x_{i} x k_{u} \quad k_{u} k_{u} x_{i} \omega}$, again with $q=1$. The sum reduces to $p_{\nu}=a_{u}$ and we are left with:

$$
C_{k_{u} k_{u}}^{(x a x) 1}=C_{x_{i} k_{u}}^{(\omega x a) a_{u}} C_{a_{u} k_{u}}^{(\omega a x) x_{i}} \mathrm{~F}_{a_{u} 1}\left[\begin{array}{ll}
x_{i} & x_{i} \\
k & k
\end{array}\right] \neq 0
$$

Again all terms on the r.h.s. have to be nonzero.

\subsection{Mixed boundaries}

Define the boundary condition label $\mu$ as $\mu=\left(1, \frac{q}{2}\right)$, i.e. the first node of the A-diagram and the upper end node of the D-diagram in (13). Consider (19) with $\mu_{\mu \omega}^{\mu_{u} \omega_{0} \omega \omega_{o \omega} \mu_{u} \mu}$ and $q_{\epsilon}=1$. The sum on the r.h.s. reduces to $p_{\nu}=\mu_{u}$ and we are left with:

$$
C_{\omega_{o} \omega_{o}}^{(\omega \omega \omega) 1}=\left(C_{\mu_{u} \omega_{o}}^{(\mu \omega \omega) \mu_{u}}\right)^{2} \mathrm{~F}_{\mu_{u} 1}\left[\begin{array}{ll}
\omega_{o} & \omega_{o} \\
\mu_{u} & \mu_{u}
\end{array}\right]
$$

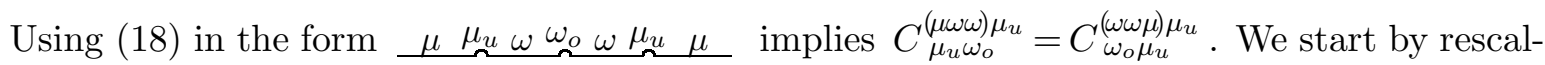
$\operatorname{ing} \psi_{\omega_{o}}^{(\omega \omega)}$ such that:

$$
C_{\omega_{o} \mu_{u}}^{(\omega \omega \mu) \mu_{u}}=1
$$

From (22) it now follows that

$$
C_{\omega_{o} \omega_{o}}^{(\omega \omega \omega) 1}=A \quad \text { where } A=\mathrm{F}_{\mu_{u} 1}\left[\begin{array}{cc}
\mu_{u} & \mu_{u} \\
\omega_{o} & \omega_{o}
\end{array}\right]
$$

defining the constant $A$. We use the freedom to rescale $\psi_{x_{o}}^{(\omega x)}$ to fix $C_{\omega_{o} x_{o}}^{(\omega \omega x)}=A$ for any i-type boundary $x$.

Let $a$ be an n-type boundary. Taking $\underbrace{\omega \omega_{o} \omega \mu_{u \mu} \ell_{u} a a_{u} \omega}$ and $q_{\epsilon}=a_{u}$ the sum reduces to $p_{\nu}=\mu_{u}$ and we see, using (23):

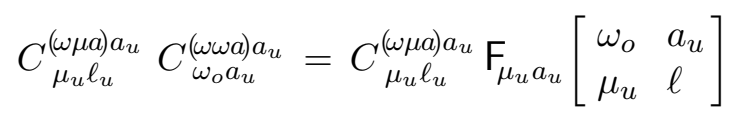

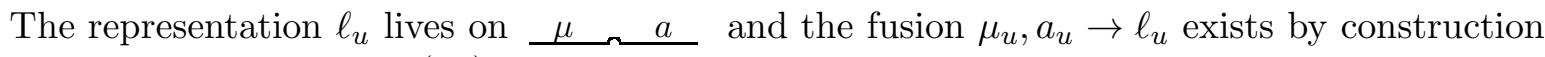
of the field content. $C_{\mu_{u} \ell_{u}}^{(\omega \mu a) a_{u}}$ is nonzero (see (21)) and the F-matrix element has to be independant of the specific choice of $\ell_{u}$ we make. We denote this F-matrix element with $B_{a}$ :

$$
B_{a}=\mathrm{F}_{\mu_{u} a_{u}}\left[\begin{array}{ll}
\omega_{o} & a_{u} \\
\mu_{u} & \ell
\end{array}\right] \quad \text { where } \underbrace{\mu \ell_{u}} \quad \underbrace{\ell_{1}}
$$


Comparing to (25) we see $C_{\omega_{o} a_{u}}^{(\omega \omega a) a_{u}}=B_{a}$. It is also useful to define a constant $C_{x}$ for any boundary $x$ as follows:

$$
x \text { of i-type: } \quad C_{x}=\mathrm{F}_{x_{e} 1}\left[\begin{array}{cc}
\omega_{o} & \omega_{o} \\
x_{o} & x_{o}
\end{array}\right] \quad x \text { of n-type: } \quad C_{x}=\mathrm{F}_{x_{u} 1}\left[\begin{array}{cc}
\omega_{o} & \omega_{o} \\
x_{u} & x_{u}
\end{array}\right]
$$

From (19) with $\omega \omega_{o} \omega \omega_{o} \omega a_{u} a a_{u} \omega, q_{\epsilon}=a_{u}$ and $p_{\nu}=1$ we get the following identity for any n-type boundary $a$ :

$$
C_{a} \cdot B_{a}=\frac{A}{B_{a}}
$$

Let $x$ and $y$ be two boundaries s.t. one is of n-type. Then by rescaling $\psi_{\ell_{u}}^{(x y)}$ for $x \neq \omega$ and $\ell \neq 1$ we fix $C_{x_{\alpha} \ell_{u}}^{(\omega x y) y_{\beta}}=1$ where $\alpha, \beta$ stand for the labels $e$ or $u$, as applicable for the present boundary conditions. Let $x$ be an i-type boundary. We can use (19) to calculate the structure constants involving $x_{o}$ instead of $x_{e}$ :

$$
C_{x_{o} \ell_{u}}^{(\omega x a) a_{u}}=\frac{A}{B_{a}} \mathrm{~F}_{x_{e} a_{u}}\left[\begin{array}{cc}
\omega_{o} & a_{u} \\
x_{o} & \ell
\end{array}\right] \quad C_{a_{u} \ell_{u}}^{(\omega a x) x_{o}}=\frac{B_{a}}{A} \mathrm{~F}_{a_{u} x_{o}}\left[\begin{array}{ll}
\omega_{o} & x_{e} \\
a_{u} & \ell
\end{array}\right]
$$

Using (19) and the identities in appendix B one can verify that the definitions above give rise to symmetrical structure constants, i.e. $C_{x_{\alpha} \ell_{u}}^{(\omega x y) y_{\gamma}}=C_{\ell_{u} x_{\alpha}}^{(y x w) y_{\gamma}}$ where at least one of $x, y$ is of n-type.

\section{3 i-type boundaries}

Let $x, y$ be i-type boundaries. Consider the structure constant $C_{x_{\alpha} \ell_{\beta}}^{(\omega x y) y_{\gamma}}$ where the labels $\alpha, \beta, \gamma$ are either $e$ or $o$ (as $u$ cannot occur in this situation). We will now try to regauge the boundary fields such that the following rule holds:

The structure constant $C_{x_{\alpha} \ell_{\beta}}^{(\omega x) y_{\gamma}}$ can be nonzero only if $\{\alpha, \beta, \gamma\}$ is one of the (unordered) sets $\{e, e, e\}$ or $\{e, o, o\}$.

Suppose that the representation $\ell$ on $x \_y$ occurs with multiplicity one. Recall from the analysis of the D-series boundary field content in section 3.3 that then the above rule is automatically true, since e.g. if $\ell$ gets label $e$ then it occurs in the fusion of $x_{e}, y_{e}$ or $x_{o}, y_{o}$ but not in the fusion of $x_{e}, y_{o}$ and $x_{o}, y_{e}$.

If the representation $\ell$ occurs with multiplicity two, we can use the freedom to form linear combinations of the two fields to make the above rule true. This is explained in more detail below.

Let $\ell$ be a representation that occurs with multiplicity two. Let $\psi_{\ell_{e}}^{(x y)}$ and $\psi_{\ell_{o}}^{(x y)}$ be the two fields. Recall that we normalised the two-point functions in such a way that only $\left\langle\psi_{\ell_{e}}^{(x y)} \psi_{\ell_{e}}^{(y x)}\right\rangle$ and $\left\langle\psi_{\ell_{o}}^{(x y)} \psi_{\ell_{o}}^{(y x)}\right\rangle$ are nonzero. When taking linear combinations of the primary fields one has to preserve this condition, or the form of the sewing constraints would change. One gets the following constraints:

$$
\left\langle\psi_{\ell_{e}}^{(x y)} \psi_{\ell_{o}}^{(y x)}\right\rangle=0 \quad, \quad\left\langle\psi_{\ell_{o}}^{(x y)} \psi_{\ell_{e}}^{(y x)}\right\rangle=0
$$


Now consider the following change of basis:

$$
\left(\begin{array}{l}
\psi_{\ell_{e}}^{(x y)} \\
\psi_{\ell_{o}}^{(x y)}
\end{array}\right)_{\text {new }}=\left(\begin{array}{ll}
a & b \\
c & d
\end{array}\right)\left(\begin{array}{c}
\psi_{\ell_{e}}^{(x y)} \\
\psi_{\ell_{o}}^{(x y)}
\end{array}\right)_{\text {old }}, \quad\left(\begin{array}{c}
\psi_{\ell_{e}}^{(y x)} \\
\psi_{\ell_{o}}^{(y x)}
\end{array}\right)_{\text {new }}=\left(\begin{array}{cc}
\tilde{a} & \tilde{b} \\
\tilde{c} & \tilde{d}
\end{array}\right)\left(\begin{array}{l}
\psi_{\ell_{e}}^{(y x)} \\
\psi_{\ell_{o}}^{(y x)}
\end{array}\right)_{\text {old }}
$$

where the two matrices are invertible. The constraints (30) amount to:

$$
a \tilde{c} \cdot C_{\ell_{e} \ell_{e}, \text { old }}^{(x y y) 1}+b \tilde{d} \cdot C_{\ell_{o} \ell_{o}, \text { old }}^{(x y x) 1}=0 \quad c \tilde{a} \cdot C_{\ell_{e} \ell_{e}, \text { old }}^{(x y x) 1}+d \tilde{b} \cdot C_{\ell_{o} \ell_{o}, \text { old }}^{(x y x) 1}=0
$$

We see that we can choose the new basis of $\psi^{(x y)}$ arbitrarily and the above condition fixes the direction, but not the length of the new basis vectors for $\psi^{(y x)}$.

This change of basis can be used to bring the following $2 \times 2-$ matrix composed of structure constants to diagonal form:

$$
\left(\begin{array}{cc}
C_{x_{e} \ell_{e}}^{(\omega x y) y_{e}} & C_{x_{e} \ell_{e}}^{(\omega x y) y_{o}} \\
C_{x_{e} \ell_{o}}^{(\omega x y) y_{e}} & C_{x_{e} \ell_{o}}^{(\omega x y)}
\end{array}\right) \longrightarrow\left(\begin{array}{cc}
* & 0 \\
0 & *
\end{array}\right)
$$

We now redefined the structure constants $C_{x_{\alpha} \ell_{\beta}}^{(\omega x) y_{\gamma}}$ in such a way that they obey our desired coupling relations $\{e, e, e\}$ or $\{e, o, o\}$.

The change of basis fixes the new $\psi_{\ell_{e}}^{(y x)}$ and $\psi_{\ell_{0}}^{(y x)}$ up to rescaling. However they do already obey the odd/even-coupling relation. To see this consider (19) in the form:

$$
\begin{aligned}
& \underbrace{\omega}_{\quad x_{e} \ell_{e} \ell_{\ell_{0}} x_{e} x_{e} \omega}, q_{\epsilon}=1 \\
& \quad C_{\ell_{e} \ell_{o}}^{(x y x) 1}=C_{x_{e} \ell_{e}}^{(\omega x y) y_{e}} C_{y_{e} \ell_{o}}^{(\omega y x) x_{e}} F_{y_{e} 1}\left[\begin{array}{ll}
\ell & \ell \\
x_{e} & x_{e}
\end{array}\right]
\end{aligned}
$$

The 1.h.s. of this equation is zero and on the r.h.s. both $C_{x_{e} \ell_{e}}^{(\omega x y) y_{e}}$ and the F-matrix entry are nonzero (this can be seen from evaluating $C_{\ell_{e} \ell_{e}}^{(x y x) 1} \neq 0$ instead of $C_{\ell_{e} \ell_{o}}^{(x y x) 1}=0$ in (34)). Hence $C_{y_{e} k_{o}}^{(\omega y x) x_{e}}=0$ as we said. (82):

From $C_{\omega_{o} x_{o}}^{(\omega \omega x) x_{e}}=A$ in the previous section we can determine $C_{\omega_{o} x_{e}}^{(\omega \omega x) x_{o}}$ by using (19) and

$$
\begin{aligned}
& \underbrace{\underbrace{\omega} \omega_{o} \omega \omega_{o} \omega x_{e} x} x_{e}^{\omega_{e} \omega}, q_{\epsilon}=x_{o} \\
& \quad \Rightarrow \quad C_{\omega_{o} x_{e}}^{(\omega \omega x) x_{o}}=\frac{C_{\omega_{o} \omega_{o}}^{(\omega \omega \omega) 1}}{C_{\omega_{o} x_{o}}^{\left(\omega \omega x x_{e}\right.}} \cdot F_{1 x_{o}}\left[\begin{array}{ll}
\omega_{o} & x_{e} \\
\omega_{o} & x_{e}
\end{array}\right]=\frac{1}{C_{x}}
\end{aligned}
$$

Next we fix the form of $C_{x_{\alpha} \ell_{\beta}}^{(\omega x y) y_{\gamma}}$. For $\ell \neq 1, x \neq \omega$ we rescale $\psi_{\ell_{e}}^{(x y)}$ and $\psi_{\ell_{0}}^{(x y)}$ s.t.:

$$
C_{x_{o} \ell_{e}}^{(\omega x y) y_{o}}=\mathrm{F}_{x_{e} y_{o}}\left[\begin{array}{ll}
\omega_{o} & y_{e} \\
x_{o} & \ell
\end{array}\right] \quad C_{x_{e} \ell_{o}}^{(\omega x y) y_{o}}= \begin{cases}1 & \text { if } x \leq y \\
\mathrm{~F}_{x_{o} y_{o}}\left[\begin{array}{ll}
\omega_{o} & y_{e} \\
x_{e} & \ell
\end{array}\right] & \text { if } x>y\end{cases}
$$

One can verify that for $\ell=1$ indeed $C_{x_{0} 1}^{(\omega x x) x_{o}}=1$ and that for $x=\omega$ the above reduces to the expressions for $C^{(\omega \omega x)}$ given before.

As in the case of mixed boundaries one can now check that the definitions in (36) give rise to reflection symmetric structure constants, but this time there is an exception for $x=y$ : In 
this case some of the structure constants are antisymmetric under reflection. As opposed to the A-series, for the D-series it is in general impossible to find a gauge in which all boundary structure constants have reflection symmetry. To see this consider (19) in the form:

$$
\begin{aligned}
& \underbrace{\omega x_{e} x \ell_{0}} \underbrace{x_{e} \omega \omega_{0} \omega} \\
& \Rightarrow \quad C_{\ell_{o} x_{e}}^{(x x \omega) x_{o}} C_{x_{e} x_{o}}^{(\omega x \omega) \omega_{o}}=C_{x_{e} \ell_{o}}^{(\omega x x) x_{o}} C_{x_{o} x_{e}}^{(\omega x \omega) \omega_{o}} F_{x_{o} x_{o}}\left[\begin{array}{ll}
x_{e} & \omega_{o} \\
\ell & x_{e}
\end{array}\right]
\end{aligned}
$$

Eqn. (81) forces the F-matrix entry in (37) to square to one. But in general it does take the values \pm 1 , depending on $\ell_{o}$. So no matter how we choose $C_{x_{e} x_{o}}^{(\omega x \omega) \omega_{o}}$ and $C_{x_{o} x_{e}}^{(\omega x \omega) \omega_{o}}$, in general we cannot avoid that if $C_{x_{e} \ell_{o}}^{(\omega x x) x_{o}}$ is symmetric for some values of $\ell_{o}$, it will be antisymmetric for others.

One can verify that (36) implies that for even fields alone we get a solution that resembles the A-series, i.e. $C_{x_{e} \ell_{e}}^{(\omega x y) y_{e}}=1$, whereas the various structure constants involving odd fields are:

$$
\begin{array}{ll}
\text { For } x \leq y: & C_{x_{e} \ell_{o}}^{(\omega x y) y_{o}}=1 ; \quad C_{x_{o} \ell_{e}}^{(\omega x y) y_{o}}=\mathrm{F}_{x_{e} y_{o}}\left[\begin{array}{ll}
\omega_{o} & y_{e} \\
x_{o} & \ell
\end{array}\right] ; \\
& C_{x_{o} \ell_{o}}^{(\omega x y) y_{e}}=A \cdot \mathrm{F}_{x_{e} y_{e}}\left[\begin{array}{ll}
\omega_{o} & y_{o} \\
x_{o} & \ell
\end{array}\right] \cdot C_{y} \\
\text { For } x>y: & C_{x_{e} \ell_{o}}^{(\omega x y) y_{o}}=\mathrm{F}_{x_{o} y_{o}}\left[\begin{array}{ll}
\omega_{o} & y_{e} \\
x_{e} & \ell
\end{array}\right] ; \quad C_{x_{o} \ell_{e}}^{(\omega x y) y_{o}}=\mathrm{F}_{x_{e} y_{o}}\left[\begin{array}{ll}
\omega_{o} & y_{e} \\
x_{o} & \ell
\end{array}\right] ; \\
& C_{x_{o} \ell_{o}}^{(\omega x y) y_{e}}=A \cdot C_{x}
\end{array}
$$

\subsection{General boundary structure constants}

Consider (19) in the form

$$
\begin{aligned}
& \underbrace{\omega x_{\alpha x} i_{r} y j_{s} z z_{\gamma} \omega} \underbrace{x_{i}=k_{t}} \\
& \quad \Rightarrow \quad C_{i_{r} j_{s}}^{(x y z) k_{t}}=\sum_{\beta} \frac{C_{x_{\alpha} i_{r}}^{(\omega x y) y_{\beta}} C_{y_{\beta} j_{s}}^{(\omega y z) z_{\gamma}}}{C_{x_{\alpha} k_{t}}^{(\omega x z) z_{\gamma}}} F_{y_{\beta} k}\left[\begin{array}{ll}
x_{\alpha} & z_{\gamma} \\
i & j
\end{array}\right]
\end{aligned}
$$

where the set $\{\alpha, t, \gamma\}$ has to be one of $\{e, e, e\},\{e, o, o\}$ or $\{u, *, *\}$. Since all constants that can occur on the r.h.s. have been computed in the previous two sections, the general boundary structure constants can be obtained from (39).

Suppose all boundaries are of i-type. Then choosing $\alpha=e$ and $\gamma=t$ reduces the sum in (39) to $\beta=r$.In the numerator on the r.h.s. we now see the boundary structure constant $C_{y_{r} j_{s}}^{(\omega y z)}$, which obeys the even/odd coupling rule as seen in section 4.3. This implies that the even/odd coupling rule extends to all boundary structure constants, i.e. $C_{i_{r} j_{s}}^{(x y z) k_{t}}$ can be nonzero only if $\{r, s, t\}$ is one of the sets $\{e, e, e\},\{e, o, o\}$ or $\{u, *, *\}$.

We can also investigate the behaviour of the boundary structure constants under reflection, i.e. given $C_{i_{r} j_{s}}^{(x y z) k_{t}}$, what is $C_{j_{s} i_{r}}^{(z y y) k_{t}}$ ? Using (18) we check that for $C_{x_{\alpha} \ell_{\beta}}^{(\omega x y) y_{\gamma}}$ reflection symmetry is equivalent to $\epsilon=1$ in the following equation:

$$
C_{x_{\alpha} \ell_{\beta}}^{(\omega x y) y_{\gamma}}=\epsilon \cdot \frac{C_{x_{\alpha} x_{\alpha}}^{(\omega x \omega) 1}}{C_{y_{\gamma} y_{\gamma}}^{(\omega y \omega) 1}} C_{y_{\gamma} \ell_{\beta}}^{(\omega y x) x_{\alpha}}
$$


As remarked in the previous two sections, using the explicit form of the structure constants derived there, we see that $\epsilon=1$ in all cases except for one, and that is $\beta=o$ and $x=y$, where we can have $\epsilon= \pm 1$. This can be made more precise in the light of $(\sqrt{37})$, if we define:

$$
\epsilon\left(x, x, \ell_{o}\right)=\mathrm{F}_{x_{o} x_{o}}\left[\begin{array}{ll}
x_{e} & \omega_{o} \\
\ell & x_{e}
\end{array}\right] \text { and } \epsilon\left(x, y, \ell_{\beta}\right)=1 \text { in all other cases }
$$

Then (40) holds with $\epsilon=\epsilon\left(x, y, \ell_{i}\right)$ for all $x, y, \ell_{i}$ and we can use this relation to evaluate the reflection property of the general boundary structure constants obtained in (39). We get:

$$
C_{i_{r} j_{s}}^{\left(x y z k_{t}\right.}=\frac{\epsilon\left(x, y, i_{r}\right) \epsilon\left(y, z, j_{s}\right)}{\epsilon\left(x, z, k_{t}\right)} \cdot C_{j_{s} i_{r}}^{(z y x) k_{t}}
$$

\subsection{Collection of results}

We found: Any solution to the boundary four-point constraint (任) for a given D-series field content can be brought to the same form by regauging the fields, i.e. if there is a solution, it is essentially unique.

In the gauge we chose, there are two conditions that have to be satisfied before a given fusion $\psi_{i_{\alpha}}^{(x y)} \psi_{j_{\beta}}^{(y z)} \rightarrow \psi_{k_{\gamma}}^{(x z)}$ can exist. First the according Verlinde fusion number $\mathrm{N}_{i j}{ }^{k}$ has to be nonzero and second the $e / u / o$-labels $\{\alpha, \beta, \gamma\}$ have to be one of the sets $\{u, *, *\},\{e, e, e\}$ or $\{e, o, o\}$ (where $*$ stands for any label). The assignment of the $e / o / u$-labels is described in section 3.3. From this even/odd-coupling rule we see immediately that the boundary structure constants involving only i-type boundaries have a $\mathbb{Z}_{2}$-symmetry of the form $\psi_{\ell_{e}}^{(x y)} \rightarrow \psi_{\ell_{e}}^{(x y)}$ and $\psi_{\ell_{0}}^{(x y)} \rightarrow-\psi_{\ell_{0}}^{(x y)}$.

There are two boundary conditions which we have endowed with a special name. $\omega$ stands for the boundary condition associated to the $(1,1)$ pair of nodes in the diagram (13) and $\mu$ for $\left(1, \frac{q}{2}\right)$. The boundary structure constants take the following form:

- Two-point functions with $\omega$ (the constants $A$ and $C_{x}$ are defined in (24) and (27)):

$$
C_{x_{u} x_{u}}^{(\omega x \omega) 1}=1 \quad C_{x_{e} x_{e}}^{(\omega x \omega) 1}=1 \quad C_{x_{o} x_{o}}^{(\omega x \omega) 1}=A \cdot C_{x}
$$

- For $x$ any type and $y$ of n-type $\left(B_{y}\right.$ is defined in (26)):

$$
C_{x_{u} \ell_{u}}^{(\omega x y) y_{u}}=1 \quad C_{x_{e} \ell_{u}}^{(\omega x y) y_{u}}=1 \quad C_{x_{o} \ell_{u}}^{(\omega x y) y_{u}}=\frac{A}{B_{y}} \mathrm{~F}_{x_{e} y_{u}}\left[\begin{array}{cc}
\omega_{o} & y_{u} \\
x_{o} & \ell
\end{array}\right]
$$

- For $x, y$ of i-type and $x \leq y$ (the ordering is defined in section 3.4):

$$
\begin{aligned}
C_{x_{e} \ell_{e}}^{(\omega x y) y_{e}} & =1 & C_{x_{e} \ell_{o}}^{(\omega x y) y_{o}} & =1 \\
C_{x_{o} \ell_{e}}^{(\omega x y) y_{o}} & =\mathrm{F}_{x_{e} y_{o}}\left[\begin{array}{cc}
\omega_{o} & y_{e} \\
x_{0} & \ell
\end{array}\right] & C_{x_{o} \ell_{o}}^{(\omega x y) y_{e}} & =A \cdot C_{y} \cdot F_{x_{e} y_{e}}\left[\begin{array}{ll}
\omega_{o} & y_{o} \\
x_{o} & \ell
\end{array}\right]
\end{aligned}
$$

All other cases can be obtained from this list by using (40) with $\epsilon=1$ (since the case $x=y$ is covered in the list), with results listed in (29), (38).

For the general boundary structure constants we distinguish two cases. First, for $x, z$ of n-type and $y$ of i-type the sum over $\beta$ in (39) has to be carried out and we get:

$$
C_{i_{u} j_{u}}^{(x y z) k_{u}}=\mathrm{F}_{y_{e} k}\left[\begin{array}{ll}
x_{u} & z_{u} \\
i & j
\end{array}\right]+\frac{B_{x}}{B_{z}} \cdot \mathrm{F}_{x_{u} y_{o}}\left[\begin{array}{ll}
y_{e} & \omega_{o} \\
i & x_{u}
\end{array}\right] \mathrm{F}_{y_{e} z_{u}}\left[\begin{array}{ll}
z_{u} & \omega_{o} \\
j & y_{o}
\end{array}\right] \mathrm{F}_{y_{o} k}\left[\begin{array}{ll}
x_{u} & z_{u} \\
i & j
\end{array}\right]
$$


In all other cases the sum reduces to one term with the result:

$$
C_{i_{r} j_{s}}^{(x y z) k_{t}}=\frac{C_{x_{\alpha} i_{r}}^{(\omega x y) y_{\beta}} C_{y_{\beta} j_{s}}^{(\omega y z) z_{\gamma}}}{C_{x_{\alpha} k_{t}}^{(\omega x z) z_{\gamma}}} \mathrm{F}_{y_{\beta} k}\left[\begin{array}{ll}
x_{\alpha} & z_{\gamma} \\
i & j
\end{array}\right]
$$

The r.h.s. does not depend on the specific choice of $\alpha, \beta, \gamma$ as long as the combinations $\{\alpha, r, \beta\}$, $\{\beta, s, \gamma\}$ and $\{\alpha, t, \gamma\}$ are allowed by the even/odd coupling rule. The structure constant in the denominator is then automatically nonzero (see section 4.1). The boundary structure constants are either symmetric or anti-symmetric under reflection, the precise behaviour is given in eqn. (42).

Note in particular that if $x, y, z$ are all of n-type or if the fields $i_{r}, j_{s}, k_{t}$ are even, the solution takes the same form as in the A-series (see eqn. (31) in [15]):

$$
C_{i_{e} j_{e}}^{(x y z) k_{e}}=\mathrm{F}_{y_{e} k}\left[\begin{array}{ll}
x_{e} & z_{e} \\
i & j
\end{array}\right] \quad \text { and } \quad C_{i_{u} j_{u}}^{(x y z) k_{u}}=\mathrm{F}_{y_{u} k}\left[\begin{array}{ll}
x_{u} & z_{u} \\
i & j
\end{array}\right]
$$

In fact this form also holds for all mixed cases with only $e / u$ field labels, except when the boundaries $x, z$ are of n-type and $y$ is of i-type, when we obtained (46).

\section{$5 \quad$ Extension to bulk theory}

The bulk-boundary couplings ${ }^{(a)} B_{i_{\alpha}}{ }^{k_{\gamma}}$ can be determined by analysing the sewing constraint (5) in a rewritten form using the assumption that all $C_{i_{\alpha} i_{\alpha}}^{(x y y) 1}$ are nonzero for fields present in the model (see section (4):

$$
\begin{gathered}
\sum_{\delta}{ }^{(b)} B_{i_{\alpha}}{ }^{\ell_{\delta}} C_{p_{\nu} \ell_{\delta}}^{(a b b) q_{\epsilon}}=\sum_{k, \gamma}{ }^{(a)} B_{i_{\alpha}}{ }^{k} C_{k_{\gamma} p_{\nu}}^{(a a b) q_{\epsilon}} \cdot \sum_{m} e^{i \pi\left(2 h_{m}-2 h_{i}-h_{p}-h_{q}+\frac{1}{2}\left(h_{k}+h_{\ell}\right)\right)} \\
\cdot F_{k m}\left[\begin{array}{cc}
\bar{c} & q \\
i & p
\end{array}\right] \mathrm{F}_{m \ell}\left[\begin{array}{cc}
i & \bar{\imath} \\
p & q
\end{array}\right]
\end{gathered}
$$

Suppose now there is a boundary condition $a$ and a bulk field $i_{\alpha}$ s.t. $i_{\alpha}$ does not couple to any boundary field on the boundary $a$, i.e. ${ }^{(a)} B_{i_{\alpha}}{ }^{k_{\gamma}}=0$ for all $k_{\gamma}$ with $n_{k a}{ }^{a} \neq 0$. Then, roughly speaking (this is discussed in more detail below), since the r.h.s. of (49) is now zero, all $B$ 's involving the bulk field $i_{\alpha}$ are zero. Since any n-point function has a limit in which we take all bulk fields to the boundary, and hence in this limit the factors in front of the conformal blocks contain the product of the $B$ 's of all bulk fields, any n-point function involving the field $i_{\alpha}$ is identically zero. This in turn just means that the bulk field $i_{\alpha}$ is not part of the model and can be left out.

Using this idea we can now determine the maximal bulk field content consistent with (49). This does not prove that the maximal field content has to be modular invariant, but it turns out that it is indeed the D-series bulk field content derived in 狛.

To make this more precise, the classification of boundary conditions and their field content in [9] establishes a one-to-one correspondence between boundary conditions and diagonal bulk fields. In this approach specifying the possible boundary conditions is equivalent to giving the diagonal part of the bulk partition function, and from 国 one knows which off-diagonal parts have to be added to make it modular invariant. The statement thus is that, at least of the $\mathrm{A}$ - and $\mathrm{D}$-series, the bulk field content obtained by this procedure is the maximal consistent one. 


\subsection{Bulk field content}

For an arbitrary boundary $x$ consider (49) with $a=\omega, b=x, p_{\nu}=x_{\nu}, q_{\epsilon}=x_{\epsilon}$. The $\ell_{\delta}$ sum reduces to one element and we get:

$$
\begin{aligned}
{ }^{(x)} B_{i_{\alpha}}{ }^{\ell_{\delta}} C_{x_{\nu} \ell_{\delta}}^{(\omega x x) x_{\epsilon}}=\left.{ }^{(\omega)} B_{i_{\alpha}}{ }^{1} C_{1 x_{\nu}}^{(\omega \omega x) x_{\epsilon}} \cdot(\text { F's })\right|_{k=1} \\
+\left.{ }^{(\omega)} B_{i_{\alpha}}{ }^{{ }^{\circ}} C_{\omega_{o} x_{\nu}}^{(\omega \omega x) x_{\epsilon}} \cdot(\text { F's })\right|_{k=\omega_{o}}
\end{aligned}
$$

First note that from an argument similar to (20) and (21) we see that all boundary structure constants that appear in $(50)$ are nonzero if they are allowed by fusion and the even/odd coupling rule (see section 4.1).

Suppose a bulk field $i_{\alpha}$ does not couple to the $\omega$-boundary, i.e. ${ }^{(\omega)} B_{i_{\alpha}}{ }^{1}=0$ and ${ }^{(\omega)} B_{i_{\alpha}}{ }^{\omega_{o}}=0$. Then the r.h.s. of (50) is identically zero and since the boundary structure constant on the l.h.s. is nonzero it follows that ${ }^{(a)} B_{i_{\alpha}}{ }^{k_{\gamma}}=0$ for all $a, k_{\gamma}$. As we argued before this implies that all correlators involving $i_{\alpha}$ vanish and the field can be removed from the bulk theory. Hence for any bulk field $i_{\alpha}$ we need at least one of ${ }^{(\omega)} B_{i_{\alpha}}{ }^{1}$ and ${ }^{(\omega)} B_{i_{\alpha}}{ }^{\omega}$ o to be nonzero.

The bulk field $i_{\alpha}$ transforms in a tensor product of two representations $i \otimes \bar{\imath}$. Suppose $i_{\alpha}$ is a field with spin, i.e. $i \neq \bar{\imath}$. Then $i$ and $\bar{\imath}$ cannot fuse to the identity and hence it follows that ${ }^{(\omega)} B_{i_{\alpha}}{ }^{1}=0$. So for the field $i_{\alpha}$ to exist we need ${ }^{(\omega)} B_{i_{\alpha}}{ }^{\omega} \neq 0$. This is only possible if the fusion $i, \bar{\imath} \rightarrow \omega_{o}$ exists, i.e. only pairs with Kac-labels $i=(r, s)$ and $\bar{\imath}=(r, q-s)$ are possible. Now consider (50) with $x=\omega, \ell_{\delta}=x_{\nu}=\omega_{o}, x_{\epsilon}=1$. We obtain (compare also to (16)):

$$
{ }^{(\omega)} B_{i_{\alpha}}{ }^{\omega_{o}}={ }^{(\omega)} B_{i_{\alpha}} \omega_{o} \cdot e^{2 \pi i\left(\bar{h}_{i}-h_{i}\right)}={ }^{(\omega)} B_{i_{\alpha}} \omega_{o} \cdot e^{2 \pi i\left(\frac{p}{2}-r\right)\left(\frac{q}{2}-s\right)}
$$

For the exponential to be +1 we need $\frac{q}{2}-s$ to be even (recall that for the D-series $p$ is always odd and $q$ always even).

Next suppose that the bulk field $i_{\alpha}$ is diagonal, i.e. $i=\bar{\imath}$. Let $i$ have Kac-labels $(r, s)$. As before denote by $i^{*}$ the representation with Kac-labels $(r, q-s)$. Consider (50) with $x=\omega$, $\ell_{\delta}=1, x_{\nu}=x_{\epsilon}=\omega_{o}$. The F-sum in (49) reduces to $m=i^{*}$ and we obtain:

$$
{ }^{(\omega)} B_{i_{\alpha}}{ }^{1}={ }^{(\omega)} B_{i_{\alpha}}{ }^{1} \cdot e^{2 \pi i\left(h_{i}{ }^{*}-h_{i}-h_{\omega_{o}}\right)}=-{ }^{(\omega)} B_{i_{\alpha}}{ }^{1} \cdot e^{-i \pi s}
$$

Hence only diagonal bulk fields with representation $i=(r, s)$ where $s$ is odd are present. Recall that by convention $r$ is always odd.

Up to now we have determined which pairs of representations are possible in the bulk. Now we have to determine their multiplicities. The maximal multiplicity a bulk field can have is equal to the number of boundary fields it couples to on the $\omega$-boundary. This can be seen as follows: Suppose two bulk fields $i_{\alpha}, i_{\beta}$ in the same representation $i \otimes \bar{\imath}$ can due to the fusion rules only couple to one field $\omega_{\gamma}$ on the $\omega$-boundary. Then we can define two new bulk fields as linear combination of $i_{\alpha}, i_{\beta}$ s.t., say, ${ }^{(\omega)} B_{i_{\alpha}, \text { new }}{ }^{\omega_{\gamma}} \neq 0$ and ${ }^{(\omega)} B_{i_{\beta}}{ }^{\omega_{\gamma} \text {,new }}=0$. Now the new $i_{\beta}$ field does not couple to the $\omega$-boundary at all and can be removed from the theory. A similar argument forbids more than two bulk fields in the same representation that by fusion can couple to the two fields on the $\omega$-boundary. The only bulk-representations that allow coupling to both fields on the $\omega$-boundary is the pair $i \otimes i$ where $i$ has Kac-labels $\left(r, \frac{q}{2}\right)$ and only in this case multiplicity two can occur.

Table 2 summarizes the maximal bulk field content consistent with a $D$-type cylinder partition function. Each representation in the table occurs once for each possible fusion. The only situation in which multiplicity arises is for pairs of representations that can fuse to both 1 and $\omega_{o}$, in which case we have multiplicity two. Note that as mentioned before this can only 


\begin{tabular}{cl}
\hline$i, \bar{\imath}$ fuse to & representations \\
\hline 1 & $(i, \bar{\imath})$ with $i=\bar{\imath}=(r, s)$ where $r$ and $s$ odd \\
$\omega_{o}$ & $(i, \bar{\imath})$ with $i=(r, s), \bar{\imath}=(r, q-s)$ where $r$ odd and $s-\frac{q}{2}$ even \\
\hline
\end{tabular}

Table 2: Maximal bulk field content for D-series

happen for a diagonal bulk field with both representations of the form $\left(r, \frac{q}{2}\right)$ and when $\frac{q}{2}$ is odd, i.e. in $D_{\text {even }}$ models.

Note that the same line of argument also works for the A-series. Here the situation is much simpler, since there is only one field, i.e. the identity, on the 1-boundary. Hence only diagonal bulk fields are allowed and only multiplicity one can occur. This is precisely the A-series modular invariant bulk field content.

\section{$5.2 \quad$ Bulk-boundary couplings}

As a first step to determine the bulk-boundary couplings we will choose a basis of primary bulk fields s.t. each field couples either to 1 or to $\omega_{o}$ on the $\omega$-boundary, but not to both simultaneously.

As we have seen in the previous section, this demand is automatically enforced by the fusion rules except for diagonal bulk fields in the representation $i \otimes i$ where $i$ has Kac-labels $\left(r, \frac{q}{2}\right)$ and we are looking at a $D_{\text {even }}$ model. In this case the corresponding bulk fields have multiplicity two and similar to the argument for redefining the boundary structure constants in section 4.3 we can take linear combinations of the two bulk fields to obtain the desired coupling rule. We will denote bulk fields that couple to 1 on the $\omega$-boundary as 'even' and bulk fields that couple to the boundary field $\omega_{o}$ as 'odd'. The new bulk-boundary couplings then fulfill ${ }^{(\omega)} B_{i_{e}}{ }^{\omega}{ }_{o}=0$ for the even bulk fields $i_{e}$ and ${ }^{(\omega)} B_{i_{o}}{ }^{1}=0$ for the odd fields $i_{o}$.

We are still free to rescale all the bulk fields $i_{\alpha}$. We use this freedom to set (the odd diagonal fields are normalised differently so that the ${ }^{(x)} B_{i_{\alpha}}{ }^{1}$ represent the Pasquier algebra as seen in section 5.6):

$$
\begin{array}{ll}
{ }^{(\omega)} B_{i_{o}}{ }^{\omega}=\frac{B_{i}}{A} \cdot \frac{S_{1}{ }^{i}}{S_{1}{ }^{1}} & \text { for diagonal odd fields } i_{o}(\text { i.e. } i=\bar{\imath}) \\
{ }^{(\omega)} B_{i_{e}}{ }^{1}={ }^{(\omega)} B_{i_{o}}{ }^{\omega}=\frac{S_{1}{ }^{i}}{S_{1}{ }^{1}} \cdot e^{i \frac{\pi}{2}\left(h_{i}-\bar{h}_{i}\right)} & \text { for all other cases } \\
{ }^{(\omega)} B_{i_{o}}{ }^{1}={ }^{(\omega)} B_{i_{e}}{ }^{\omega}=0 & \text { from the coupling rule }
\end{array}
$$

With this redefinition of the fields the sum on the r.h.s. of (50) reduces to one term. Using (50) with $\epsilon=\delta$ and $k_{\gamma}=\omega_{\alpha}$ the general bulk-boundary coupling takes the form:

$$
\begin{aligned}
{ }^{(x)} B_{i_{\alpha}}{ }^{{ }_{\delta}}={ }^{(\omega)} B_{i_{\alpha}}{ }^{\omega_{\alpha}} & \cdot \sum_{m} \exp \left(i \pi\left(2 h_{m}-2 h_{i}-h_{x_{\nu}}-h_{x_{\delta}}+\frac{1}{2}\left(h_{\omega_{\alpha}}+h_{\ell}\right)\right)\right) \\
\cdot & \mathrm{F}_{\omega_{\alpha} m}\left[\begin{array}{ll}
\bar{\imath} & x_{\delta} \\
i & x_{\nu}
\end{array}\right] \mathrm{F}_{m \ell}\left[\begin{array}{ll}
i & \bar{\imath} \\
x_{\nu} & x_{\delta}
\end{array}\right] \cdot \begin{cases}1 & : \alpha=e \text { and } \delta=e \text { or } u \\
C_{x}-1 & : \alpha=o \text { and } \delta=o \\
B_{x} & : \alpha=o \text { and } \delta=u \\
0 & : \text { otherwise }\end{cases}
\end{aligned}
$$

where $\nu=e$ if $x$ is an i-type boundary and $\nu=u$ if $x$ is an n-type boundary. 
Note in particular that any bulk field can couple to a $u$-field on an n-type boundary, but for an i-type boundary an even bulk field can only couple to an even boundary field and an odd bulk field to an odd boundary field. This indicates that the $\mathbb{Z}_{2}$-symmetry of the boundary structure constants for i-type boundaries carries over to the bulk.

\subsection{Bulk structure constants}

The bulk structure constants can now be obtained from (6). Take $a=\omega$, the sum on the l.h.s. then reduces to the term where ${ }^{(\omega)} B_{m_{\rho}}{ }^{k_{\gamma}} \neq 0$, i.e. $k_{\gamma}=\omega_{\rho}$. On the r.h.s. the sum over $p_{\nu}$ and $q_{\epsilon}$ reduces to $p_{\nu}=\omega_{\alpha}, q_{\epsilon}=\omega_{\beta}$. The $r$-sum is also reduced to one element. We are left with:

$$
\begin{aligned}
& C_{i_{\alpha} j_{\beta}}{ }^{m_{\gamma}}=\exp \left(i \frac{\pi}{2}\left(h_{\omega_{\gamma}}+h_{\omega_{\alpha}}-h_{\omega_{\beta}}+2\left(h_{j}-h_{r}\right)+h_{m}-\bar{h}_{m}-h_{i}+\bar{h}_{i}-h_{j}+\bar{h}_{j}\right)\right) \\
& \cdot \frac{{ }_{(\omega)} B_{i_{\alpha}}{ }^{\omega_{\alpha}}{ }^{(\omega)} B_{j_{\beta}}{ }^{\omega_{\beta}}}{{ }^{(\omega)} B_{m_{\gamma}}{ }^{\omega_{\gamma}}} \cdot \mathrm{F}_{\mu_{u} \omega_{\gamma}}\left[\begin{array}{ll}
\mu_{u} & \mu_{u} \\
\omega_{\alpha} & \omega_{\beta}
\end{array}\right] \mathrm{F}_{\omega_{\beta} r}\left[\begin{array}{cc}
\omega_{\gamma} & \bar{\jmath} \\
\omega_{\alpha} & j
\end{array}\right] \mathrm{F}_{\omega_{\alpha} m}\left[\begin{array}{ll}
\bar{\imath} & r \\
i & j
\end{array}\right] \mathrm{F}_{r \bar{m}}\left[\begin{array}{ll}
\bar{\imath} & \bar{\jmath} \\
m & \omega_{\gamma}
\end{array}\right]
\end{aligned}
$$

where $r=j$ if $\alpha=e$ and $r=j^{*}$ if $\alpha=o$. The first F-matrix element in (55) implements an even/odd coupling rule for bulk fields, i.e. the only combinations for $\{\alpha, \beta, \gamma\}$ which can be nonzero are $\{e, e, e\}$ and $\{e, o, o\}$.

This implies that apart from other symmetries the bulk structure constants may have there is a manifest $\mathbb{Z}_{2}$-symmetry which sends even bulk fields to themselves and odd one to minus themselves. Together with section 4.5 and eqn. (54) we can now conclude that any correlator with no or only i-type boundaries is manifestly invariant under the $\mathbb{Z}_{2}$-symmetry $e \rightarrow e$ and $o \rightarrow-o$ applied to bulk and boundary simultaneously.

It is useful to know the bulk two-point functions in the present normalisation of fields. From the explicit expression in (55) we get:

$$
\begin{aligned}
C_{i_{\alpha} i_{\alpha}}{ }^{1} & =\frac{S_{1}{ }^{i}}{S_{1}{ }^{1}} & & \text { for diagonal fields } i_{\alpha} \text { (i.e. } \\
C_{i_{o} i_{o}}{ }^{1} & =A \cdot \mathrm{F}_{i 1}\left[\begin{array}{ll}
\omega_{o} & \omega_{o} \\
\bar{\imath} & \bar{\imath}
\end{array}\right] \cdot \frac{S_{1}{ }^{i}}{S_{1}{ }^{1}} \cdot(-1)^{s\left(i_{o}\right)} & & \text { for nondiagonal fields } i_{o}
\end{aligned}
$$

where $s\left(i_{\alpha}\right)=h_{i}-\bar{h}_{i} \in \mathbb{Z}$ is the spin of the bulk field $\phi_{i_{\alpha}}$.

In [14] it was shown that the minimal model bulk structure constants in the A- and Dseries are related by rational numbers, called relative structure constants. Taking the explicit expression (55) together with the normalisation (56) we find (numerical) agreement with these results. Also, for unitary models the signs of the two-point structure constants in (56) are the same as in [14], where they were shown to lead to real bulk structure constants (in the unitary case).

Note that if all bulk fields are even the solution (55) takes the same form as in the A-series:

$$
C_{i_{e} j_{e}}{ }^{m_{e}}=\left(\mathrm{F}_{m 1}\left[\begin{array}{ll}
i & i \\
j & j
\end{array}\right]\right)^{-1}
$$

Together with equations (48) for the boundary structure constants and (54) for the bulkboundary couplings this illustrates another interesting point. If one considers the even fields alone, that is all bulk and boundary fields that are invariant under the $\mathbb{Z}_{2}$-symmetry mentioned above, they form a subalgebra (as the coupling $e, e \rightarrow o$ is not allowed) and the structure constants are identical to the A-series in the following way: 
Consider the A-series boundary theory associated to the pair of diagrams $\left(A_{p-1}, A_{q-1}\right)$. In this theory consider only the boundary conditions $(\alpha, \beta)$ with $\alpha<\frac{p}{2}$ and $\beta<\frac{q}{2}$ or $\alpha>\frac{p}{2}$ and $\beta>\frac{q}{2}$ (compare to (15)) and the bulk fields $i \otimes i$ where $i$ has Kac-labels $(r, s)$ with both $r$ and $s$ odd. Then this is a closed subset of fields of the A-series theory and in the normalisation chosen its structure constants coincide with those of the invariant fields of the D-series theory $\left(A_{p-1}, D_{\frac{q}{2}+1}\right)$. This correspondence seems natural form the point of view that the invariant part of the D-diagram is an A-diagram.

\subsection{Real structure constants}

In the normalisation chosen in this paper the F-matrices are all real. This implies first of all that the boundary structure constants as given in section 4.5 are all real.

For the bulk structure constants we have seen in (57) that they are real as long as only even fields are involved. If odd fields are present consider (55) in the case $C_{i_{o} j_{o}}{ }^{m_{e}}$. The phase factors cancel and we are left with a real expression. All other cases are real as well, as can for example be seen from the three-point function $C_{i_{\alpha} j_{\beta}}{ }^{k_{\gamma}} C_{k_{\gamma} k_{\gamma}}{ }^{1}=C_{j_{\beta} k_{\gamma}}{ }^{i_{\alpha}} C_{i_{\alpha} i_{\alpha}}{ }^{1}$ together with the fact that all two-point structure constants (56) are real. Thus in the present normalisation all bulk structure constants are real, both for unitary and non-unitary models.

For the bulk-boundary couplings in (54) we can apply the identity (86) to obtain the result:

$$
\left({ }^{(x)} B_{(i \otimes \bar{\imath})_{\alpha}}{ }^{\ell_{\delta}}\right)^{*}={ }^{(x)} B_{(\bar{\imath} \otimes i)_{\alpha}}{ }^{\ell_{\delta}}
$$

This means that complex conjugation relates the bulk-boundary coupling for a bulk field of spin $s$ to that of the field of spin $-s$. In particular all bulk-boundary couplings for diagonal bulk fields are real.

It is in general not possible to choose a basis of primary fields s.t. all structure constants are real. To see this we construct a gauge invariant expression from the sewing constraints which cannot be fulfilled by real structure constants. Consider for example two diagonal bulk fields $i_{\alpha}$ and $m_{\rho}$ and a field $j_{\beta}$ with spin one. Take (6) with $k=1$ and $a=\omega$. This forces $p_{\nu}=q_{\epsilon}=\omega_{o}$ and $r=\bar{\jmath}$. We get:

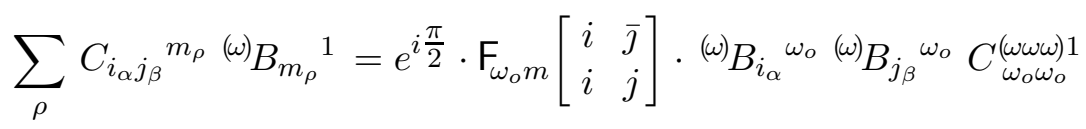

The F-matrix entry is real and will in general be nonzero, provided that all fusions are allowed. As discussed in section 5.1 the spin one field $j_{\beta}$ has to couple to $\omega_{o}$ (i.e. ${ }^{(\omega)} B_{j_{\beta}}{ }^{\omega_{o}} \neq 0$ ) and we can find a diagonal field $i_{\alpha}$ that couples to $\omega_{o}$. The boundary two-point function has to be nonzero by assumption b) in section 4. Hence the r.h.s. is nonzero and thus some of the D-series structure constants will have nonzero imaginary part if they solve the sewing constraints.

We can however consider the following non-primary basis of bulk fields to obtain real structure constants: Diagonal (even or odd) bulk fields stay as they are and for a pair $\phi_{s}, \phi_{-s}$ of nondiagonal bulk fields with spins $\pm s$ we define a new set of fields as follows:

$$
\phi^{r}=\frac{1}{2}\left(\phi_{s}+\phi_{-s}\right) \quad \phi^{i}=\frac{1}{2 i}\left(\phi_{s}-\phi_{-s}\right)
$$

The new fields $\phi^{r}$ and $\phi^{i}$ are no longer primary, but one finds that the coefficients describing their behaviour under arbitrary conformal mappings are all real. Since the OPE is determined 
from the transformation behaviour of the fields one expects the coefficients appearing in the OPE to be real as well.

One can see explicitly from (58) that all bulk-boundary couplings ${ }^{(x)} B_{\phi^{r}} \psi$ and ${ }^{(x)} B_{\phi^{i}}{ }^{\psi}$ are real and one can verify numerically that all bulk structure constants are real in the new basis as well. This holds for both unitary and non-unitary models.

\subsection{Discrete symmetries of the structure constants}

Denote the set of bulk and boundary structure constants given in (43) $-(47)$ and (53)-(55) with $A$. We see that $A$ depends explicitly on the assignment of $e / o$-labels in section 3.3 and the ordering of the boundary conditions in section 3.4 .

Denote the set of structure constants obtained by the same procedure, but with a different initial assignment of $e / o$-labels in section 3.3 as $B$. It was shown in this paper that any solution to the sewing constraints with the D-series field content can be brought to the form $A$. In particular there is a change of basis in the set of primary fields that maps $B$ to $A$.

For the primary fields in $A$ we considered a $\mathbb{Z}_{2}$-action that takes even fields to themselves and odd fields to minus themselves. This action leaves the structure constants involving no or only i-type boundaries invariant.

However a different initial $e / o$-labelling leads to a different action of $\mathbb{Z}_{2}$ on the boundary fields. The $\mathbb{Z}_{2}$-action of $B$ does via the change of basis induce a $\mathbb{Z}_{2}$-action on $A$ which is different from the original one. It follows that each distinct $e / o$-assignment results in a distinct $\mathbb{Z}_{2}$-action on $A$.

Going back to section 3.3 we see that for each i-type boundary $x \neq \omega$ we are free to choose which of the two fields living on $\_\_x$ we call $x_{e}$ and which $x_{o}$. The specific assignment made in section 3.3 was motivated on physical grounds.

There are altogether $N=2^{(p-1)(q-2) / 4-1}$ distinct assignments. Thus among other symmetries the structure constants may have, there are $N \mathbb{Z}_{2}$ 's acting on the set of primary fields that leave the subset of the structure constants in $A$ that involve no or only i-type boundary conditions invariant. One can convince oneself that the $\mathbb{Z}_{2}$ 's all act in the same way on the bulk fields but can be distinguished from their action on the boundary fields. The collection of $\mathbb{Z}_{2}$ 's just presented does not necessarily exhaust all discrete symmetries as can be seen for example in the three-states Potts model which has an additional $\mathbb{Z}_{3}$-symmetry of whose $\mathbb{Z}_{2}$ subgroups only one is contained in the above list.

\subsection{Disc partition function and Pasquier Algebra}

Up two now all constraints on the structure constants were derived in such a way that the vacuum expectation values $\langle 1\rangle_{\mathrm{UHP}}^{(x)}$ cancelled. We will next derive some constraints for the vacuum expectation values of the unit disc $\langle 1\rangle_{\mathrm{D}}^{(x)}$. Consider the two-point function $\left\langle\psi_{i_{\alpha}}^{(x y)} \psi_{i_{\alpha}}^{(y x)}\right\rangle$ on the unit disc. Taking different limits leads to the constraint:

$$
C_{i_{\alpha} i_{\alpha}}^{(x y x) 1}\langle 1\rangle_{\mathrm{D}}^{(x)}=C_{i_{\alpha} i_{\alpha}}^{(y x y) 1}\langle 1\rangle_{\mathrm{D}}^{(y)}
$$

The vacuum expectation values $\langle 1\rangle_{\mathrm{D}}^{(x)},\langle 1\rangle_{\mathrm{D}}^{(y)}$ can be interpreted as the partition function of the unit disc with different boundary conditions. This expression thus relates the partition functions of different boundary conditions in the same geometry. The relation (61) remains true for different (genus zero) geometries, but the actual values of the partition functions do depend on the shape of the system (see e.g. [20]). For example the partition function for a disc of radius $r$ is given by $\langle 1\rangle_{\mathrm{D}}^{(x)} \cdot r^{c / 6}$, where $c$ is the central charge [20]. 
Substituting the explicit boundary two-point functions into (61) we arrive at the following expressions (let $x, y$ be i-type boundaries and $a, b$ be n-type boundaries):

$$
\frac{\langle 1\rangle_{\mathrm{D}}^{(x)}}{S_{1}{ }^{x_{e}}}=\frac{\langle 1\rangle_{\mathrm{D}}^{(y)}}{S_{1}{ }^{y_{e}}} \quad \frac{\langle 1\rangle_{\mathrm{D}}^{(x)}}{S_{1}^{x_{e}}}=2 \cdot \frac{\langle 1\rangle_{\mathrm{D}}^{(a)}}{S_{1}{ }^{a_{u}}} \quad \frac{\langle 1\rangle_{\mathrm{D}}^{(a)}}{S_{1}^{a_{u}}}=\frac{\langle 1\rangle_{\mathrm{D}}^{(b)}}{S_{1}^{b_{u}}}
$$

In [9] the matrices $n_{i}$ that descibe the boundary field content are simultaneously diagonalised in the following way:

$$
n_{i x}^{y}=\sum_{j_{\beta} \text { diag. }} \frac{S_{i}^{j}}{S_{1}^{j}} \cdot \psi_{x, j_{\beta}}\left(\psi_{y, j_{\beta}}\right)^{*}
$$

where the sum runs over all (even or odd) diagonal bulk fields and the vectors $\psi_{x}$ form a complete orthonormal basis.

Replicating the line of argument in section 8 of [15] in the present notation we get the following expression for the matrices $n_{i}$ :

$$
n_{i x}{ }^{y}=\sum_{j_{\beta} \text { diag. }} \frac{S_{i}^{j}}{S_{1}{ }^{j}} \cdot \frac{S_{1}{ }^{j}}{C_{j_{\beta} j_{\beta}}{ }^{1}\langle 0 \mid 0\rangle}{ }^{(x)} B_{j_{\beta}}{ }^{1}\langle 1\rangle_{\mathrm{D}}^{(x)}{ }^{(y)} B_{j_{\beta}}{ }^{1}\langle 1\rangle_{\mathrm{D}}^{(y)}
$$

Using (56) and normalising the bulk vacuum:

$$
\langle 0 \mid 0\rangle=S_{1}^{1}
$$

we find the following relation between the bulk-boundary couplings and the vectors $\psi_{x}$ :

$$
\psi_{x, j_{\beta}}={ }^{(x)} B_{j_{\beta}}{ }^{1}\langle 1\rangle_{\mathrm{D}}^{(x)}
$$

By evaluating (66) for $j=1$ and recalling that ${ }^{(x)} B_{1}{ }^{1}=1$ we get:

$$
\langle 1\rangle_{\mathrm{D}}^{(x)}=\psi_{x, 1}
$$

Furthermore we see that the the bulk-boundary couplings to the identity on the boundary are given by a ratio of $\psi$ 's $[9]$ :

$$
{ }^{(x)} B_{j_{\beta}}{ }^{1}=\frac{\psi_{x, j_{\beta}}}{\psi_{x, 1}}
$$

In the present normalisation the $B$ 's form one dimensional representations of an algebra, a fact that was used to classify possible boundary conditions in [6 9]. The algebra was identified as the Pasquier algebra [21] in [9]:

$$
{ }^{(x)} B_{i_{\alpha}}{ }^{1{ }^{(x)}} B_{j_{\beta}}{ }^{1}=\sum_{k_{\gamma}} M_{i_{\alpha} j_{\beta}}{ }^{k_{\gamma}} \cdot{ }^{(x)} B_{k_{\gamma}}{ }^{1}
$$

where $M_{i_{\alpha} j_{\beta}}{ }^{k_{\gamma}}=\sum_{x} \psi_{x, i_{\alpha}} \psi_{x, j_{\beta}}\left(\psi_{x, k_{\gamma}}\right)^{*} / \psi_{x, 1}$.

From the relations (67), (68) and (62) together with the constraint that the vectors $\psi_{x}$ form an orthonormal basis we get the following expression:

$$
\psi_{x, i_{\alpha}}={ }^{(x)} B_{i_{\alpha}}{ }^{1} \psi_{x, 1} \quad \psi_{x, 1}= \begin{cases}\sqrt{2} \cdot S_{1}{ }^{x_{e}} & \text { if } x \text { is of i-type } \\ \frac{1}{\sqrt{2}} \cdot S_{1}{ }^{x_{u}} & \text { if } x \text { is of n-type }\end{cases}
$$

Since the bulk-boundary couplings are known from (54) one can now check that the $\psi$ 's so defined are all real and verify numerically that they fulfill (63), with $n_{i x}^{y}$ on the l.h.s. taken from section 3.3 . 


\section{Conclusion}

The aim of this paper was to find all structure constants of the D-series minimal model boundary conformal field theory and to point out some of their properties.

The structure constants can be calculated in several steps. The basic ingredient are the fusion matrices, as all structure constants are expressed in terms of these. A procedure to obtain the fusion matrices recursively is given in [15], together with references to the original literature. The matrices can also be extracted by rescaling explicit expressions found e.g. in [17] to match the normalisation fixed in appendix A. As a first step the D-series field content of the boundary theory is computed, as described in section 3.3. Next all boundary structure constants are obtained from the results collected in section 4.5. The boundary theory is now fully determined. The extension from the boundary to the bulk recovers the familiar D-series bulk field content as the maximal set of bulk fields consistent with the boundary theory (see section 5.1). Last, the remaining two sets of structure constants, the bulk-boundary couplings and the bulk structure constants are given in (53), (54) and (55).

The boundary structure constants $C_{r_{\alpha} s_{\beta}}^{(x y z) t_{\gamma}}$ and the bulk structure constants $C_{i_{\alpha} j_{\beta}}{ }^{k_{\gamma}}$ obtained by this procedure are all real, both for unitary and non-unitary minimal models. However some of the bulk-boundary couplings ${ }^{(x)} B_{i_{\alpha}}{ }^{{ }_{\delta}}$ are imaginary. There is in general no basis of primary fields such that all structure constants real. In section 5.4 a non-primary basis of fields was presented in which all three sets of structure constants are real.

The solution described above was obtained by deriving a necessary form of the structure constants. It was shown that any solution to the sewing constraints (4)-(7) for the D-series field content can be brought to this form. So just as for the A-series it follows that the structure constants are unique up to redefinition of the fields. It was however not analytically shown that the given structure constants do indeed solve the full set of genus zero sewing constraints, since they are overdetermined and only a subset was used for the actual solution. But numerical tests carried out for several minimal models where each of the equations (14)-(7) was checked repeatedly with a different random selection of fields show no contradiction.

It was also found that a subset of the D-series structure constants have a manifest $\mathbb{Z}_{2}-$ symmetry: If no or only i-type boundaries are present in a correlator it is invariant under the change 'even' $\rightarrow$ 'even' and 'odd' $\rightarrow$ - 'odd'. This symmetry can be associated to the $\mathbb{Z}_{2}-$ symmetry of the D-diagram in the sense that the boundary conditions fall in two classes: the i-type boundaries are associated to nodes left invariant by that symmetry, and the n-type boundaries to nodes which are not. We have also seen that the even fields alone form a closed subalgebra with structure constants equal to the corresponding ones of an A-type theory.

In deriving the structure constants we have used only the Virasoro algebra, and not considered the fact that it could be part of a larger symmetry algebra, as is the case for $D_{\text {even }}$ models. From the present point of view, curious properties of the solution such as the boundary field content, the even/odd coupling rule or numerical coincidences in the structure constants, are obtained as results of the calculation, but lack an explanation in terms of the additional structure present in the theory. One can use the maximally extended chiral algebra as a starting point for the analysis of a boundary conformal field theory (see e.g. 22] and references therein) and it would be interesting to interpret the explicit results presented here in this more general framework.

In section 5.5 it was pointed out that an initial freedom in the labelling of the boundary fields actually implies that the just mentioned $\mathbb{Z}_{2}$ is part of a collection of $2^{(p-1)(q-2) / 4-1}$ $\mathbb{Z}_{2}$ 's, each acting in a distinct way on the set of primary boundary fields. This action leaves structure constants with no or only i-type boundaries invariant. 
Finally the vacuum expectation values $\langle 1\rangle_{\mathrm{D}}^{(x)}$ of the unit disc with different boundary conditions were computed and it was pointed out that in the present normalisation the bulkboundary couplings ${ }^{(x)} B_{i_{\alpha}}{ }^{1}$ form one-dimensional representations of the Pasquier algebra.

To make contact with different conventions in the literature the bulk and boundary fields may have to be redefined. The normalisations chosen in this paper can be obtained from (56) in terms of the bulk two-point functions, or from (53) in terms of the bulk-boundary couplings to the identity field on the boundary.

Acknowledgements - This work was supported by the EPSRC, the DAAD and King's College London. I am especially grateful to my supervisor G.M.T. Watts for suggesting the project, encouragement, criticism and helpful conversations. Furthermore I wish to thank V.B. Petkova, P. Ruelle and J.-B. Zuber for useful discussions.

\section{Appendix A: Derivation of the five-point constraint}

This appendix was included to illustrate how the notation and techniques for calculating with conformal blocks described in [11] were used to rederive the sewing constraints in [10].

The sewing constraints arise from taking two different limits in a given correlator where the fields approach each other or the boundary and demanding that the two sets of structure constants and conformal blocks give rise to the same function.

Conformal blocks associated to different asymptotic regimes can be transformed into each other using the fusion- or $\mathrm{F}$-matrices. The $\mathrm{F}$-matrices are defined as follows:

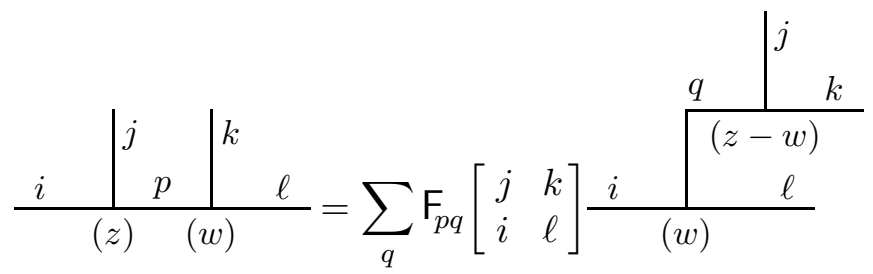

The conformal blocks appearing in (71) solve the linear differential equations associated with the (chiral) correlator $\left\langle i\left|\phi_{j}(z) \phi_{k}(w)\right| l\right\rangle$.

The normalisation convention for conformal blocks chosen in this paper is (the dots stand for a regular expression in $w)$ :

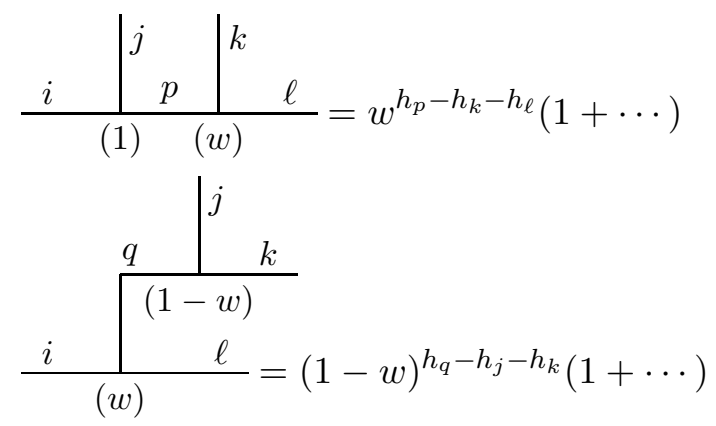

The example we consider is the derivation of the constraint resulting from taking different limits in the correlator involving two bulk fields $\phi_{i_{\alpha}}, \phi_{j_{\beta}}$ and one boundary field $\psi_{k_{\gamma}}^{(a a)}$ on the upper half plane with the boundary condition labelled $a$.

In the first limit we take the two bulk fields to the boundary and are left with a three-point function on the boundary. In the second limit we start by taking the OPE of the two bulk 
fields and then take the remaining bulk field to the boundary. Let $z=x_{z}+i y_{z}$ and $w=x_{w}+i y_{w}$. The asymptotic behaviour in the two limiting cases is then given by:

$$
\begin{aligned}
& \left\langle\phi_{i_{\alpha}}(z, \bar{z}) \phi_{j_{\beta}}(w, \bar{w}) \psi_{k_{\gamma}}^{(a a)}(x)\right\rangle
\end{aligned}
$$

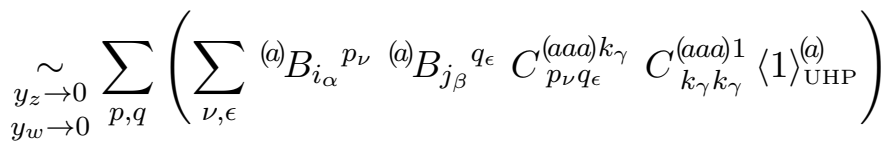

$$
\begin{aligned}
& \cdot\left(2 y_{z}\right)^{h_{p}-h_{i}-\bar{h}_{i}}\left(2 y_{w}\right)^{h_{q}-h_{j}-\bar{h}_{j}}\left(x_{z}-x_{w}\right)^{h_{k}-h_{p}-h_{q}} \\
& \cdot\left(x_{z}-x\right)^{h_{q}-h_{p}-h_{k}}\left(x_{w}-x\right)^{h_{p}-h_{k}-h_{q}}
\end{aligned}
$$

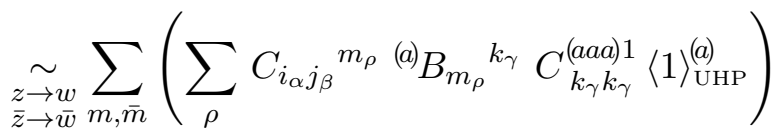

$$
\begin{aligned}
& \cdot(z-w)^{h_{m}-h_{i}-h_{j}}(\bar{z}-\bar{w})^{\bar{h}_{m}-\bar{h}_{i}-\bar{h}_{j}}\left(2 y_{w}\right)^{h_{k}-h_{m}-\bar{h}_{m}}\left(x_{w}-x\right)^{-2 h_{k}}
\end{aligned}
$$

On the other hand the correlator can be expressed as a linear combination of conformal blocks. We use two sets of conformal blocks to express the correlator in two different ways, one associated to each asymptotic behaviour in (73). We obtain the following two linear combinations:

$$
\begin{aligned}
& \left\langle\phi_{i_{\alpha}}(z, \bar{z}) \phi_{j_{\beta}}(w, \bar{w}) \psi_{k_{\gamma}}^{(a a)}(x)\right\rangle
\end{aligned}
$$

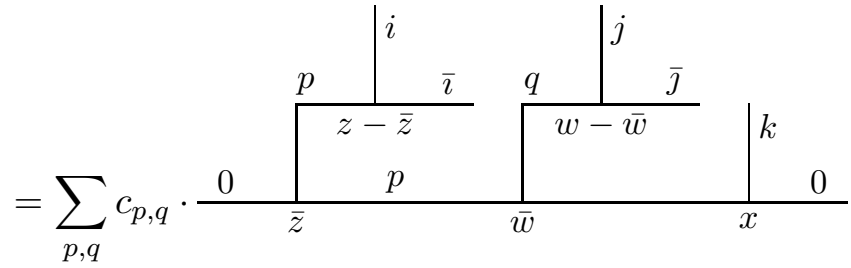

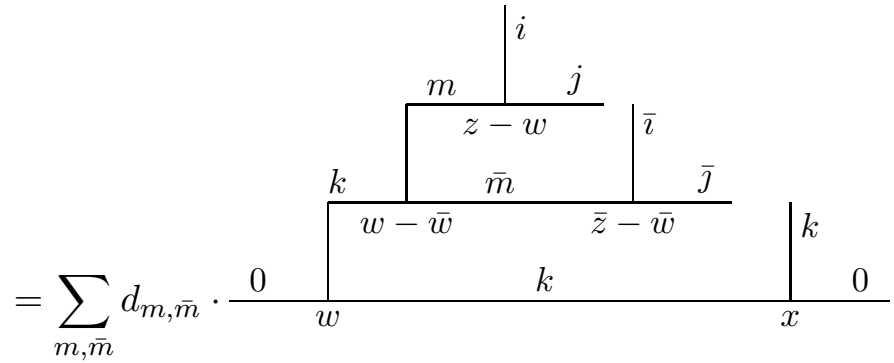

Taking the limits calculated in (73) for the exact expressions in terms of conformal blocks (74) relates the coefficients $c_{p, q}$ and $d_{m, \bar{m}}$ to the products of structure constants obtained by applying the OPE. The precise relation is:

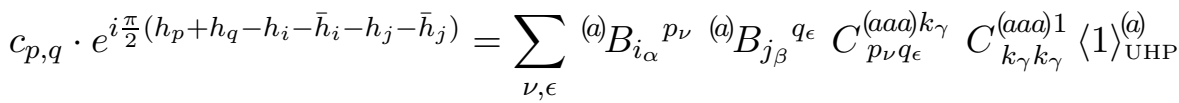

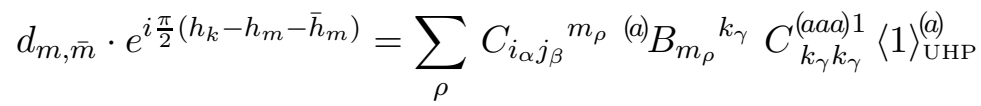

The phase factors originate from relating $z-\bar{z}=2 i y_{z}$ to $2 y_{z}$ etc. The sums over $\nu, \epsilon$ and $\rho$ in (75) take care of fields with multiplicities. Fields that transform in the same representation of the Virasoro algebra show the same asymptotic behaviour and cannot be discriminated by conformal blocks. Hence their structure constants occur as a sum in front of the according conformal block. 
The two sets of conformal blocks in (74) are related by a basis transformation. This transformation can be carried out in several steps making use of two basic moves of braiding and fusion implemented by the $\mathrm{B}-$ and $\mathrm{F}$ - matrix (see [1] for details). One possible way to perform the basis transformation is as follows:

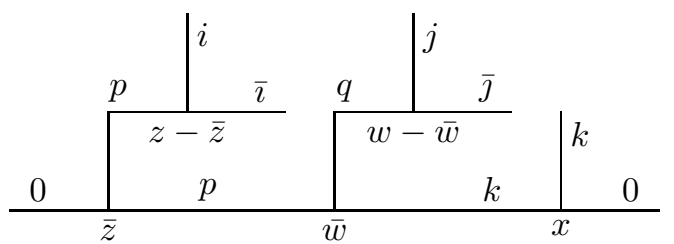

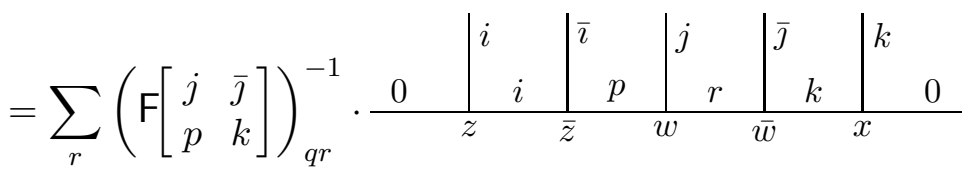

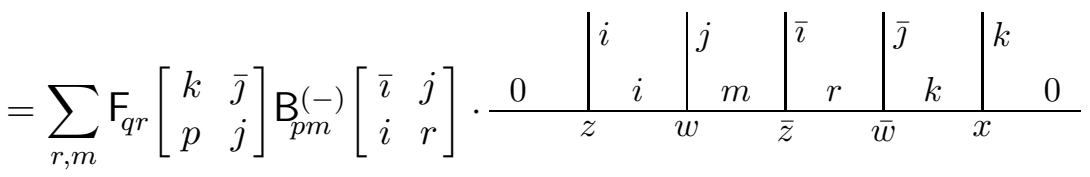

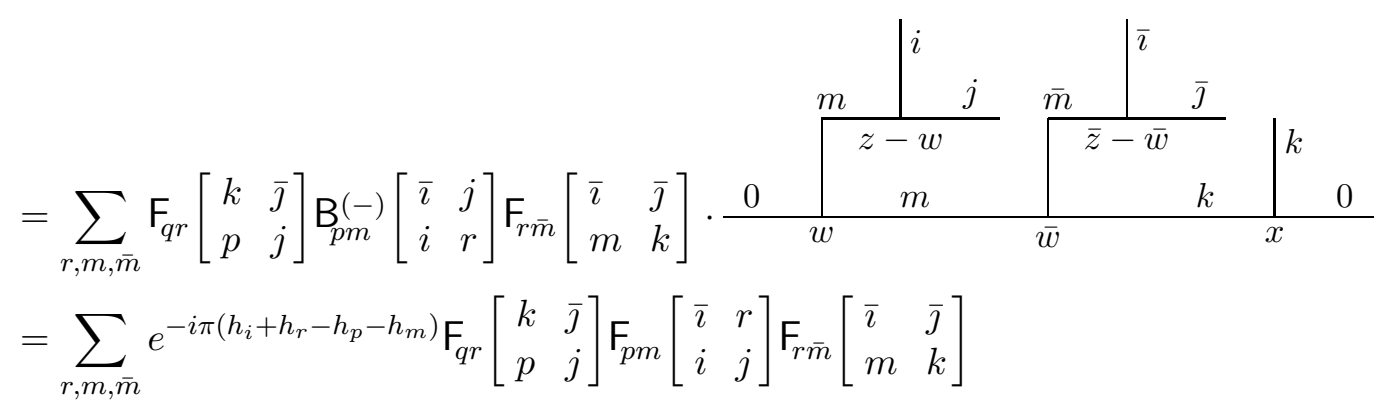

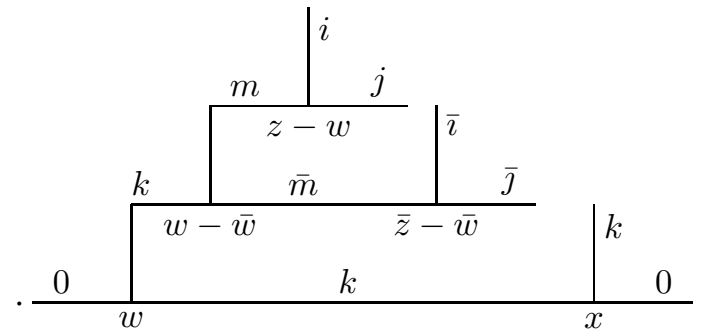

Putting (74), (75) and (76) together we recover the sewing constraint (6):

$$
\begin{aligned}
& \sum_{\rho} C_{i_{\alpha} j_{\beta}}{ }^{m_{\rho}}{ }^{(a)} B_{m_{\rho}}{ }^{k_{\gamma}} C_{k_{\gamma} k_{\gamma}}^{(a a a) 1}\langle 1\rangle_{\mathrm{UHP}}^{(a)} \\
&=\sum_{p, q}\left(\sum_{\nu, \epsilon}{ }^{(a)} B_{i_{\alpha}}{ }^{p_{\nu}}{ }^{(a)} B_{j_{\beta}}{ }^{q_{\epsilon}} C_{p_{\nu} q_{\epsilon}}^{(a a a) k_{\gamma}} C_{k_{\gamma} k_{\gamma}}^{(a a a) 1}\langle 1\rangle_{\mathrm{UHP}}^{(a)}\right) \\
& \cdot \sum_{r} e^{i \frac{\pi}{2}\left(h_{k}+h_{p}-h_{q}-2 h_{r}+h_{m}-\bar{h}_{m}-h_{i}+\bar{h}_{i}+h_{j}+\bar{h}_{j}\right)} \\
& \cdot \mathrm{F}_{q r}\left[\begin{array}{cc}
k & \bar{\jmath} \\
p & j
\end{array}\right] \mathrm{F}_{p m}\left[\begin{array}{ll}
\bar{\imath} & r \\
i & j
\end{array}\right] \mathrm{F}_{r \bar{m}}\left[\begin{array}{ll}
\bar{\imath} & \bar{\jmath} \\
m & k
\end{array}\right]
\end{aligned}
$$

The summation range for $r$ is principally over all entries in the Kac-table (quotiened by $\mathbb{Z}_{2}$ ) 
but the $\mathrm{F}$-matrix entries will only be nonzero if the following three fusions are allowed:

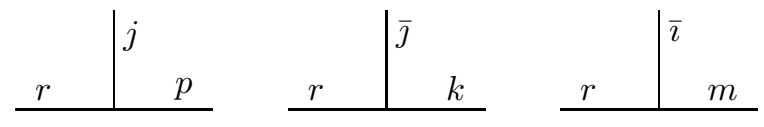

\section{Appendix B: Some F-matrix identities}

The following constitutes a collection of F-matrix identities for Virasoro minimal models used in the paper. They are either directly taken from [11] or special cases thereof. Note that for the S-matrix we have $S_{1}{ }^{i}=S_{1}{ }^{i^{*}}$ where the $*$-operation was defined as $i^{*}=(r, q-s)$ if $i$ has Kac-labels $(r, s)$.

$$
\begin{aligned}
& \mathrm{B}_{p q}^{(\epsilon)}\left[\begin{array}{ll}
j & k \\
i & \ell
\end{array}\right]=e^{i \pi \epsilon\left(h_{i}+h_{\ell}-h_{p}-h_{q}\right)} \mathrm{F}_{p q}\left[\begin{array}{ll}
j & l \\
i & k
\end{array}\right] \\
& \mathrm{F}_{p q}\left[\begin{array}{cc}
j & k \\
i & \ell
\end{array}\right]=\mathrm{F}_{p q}\left[\begin{array}{ll}
i & \ell \\
j & k
\end{array}\right]=\mathrm{F}_{p q}\left[\begin{array}{ll}
\ell & i \\
k & j
\end{array}\right] \\
& \sum_{r} F_{p r}\left[\begin{array}{ll}
b & c \\
a & d
\end{array}\right] F_{r q}\left[\begin{array}{cc}
d & c \\
a & b
\end{array}\right]=\delta_{p, q} \quad F_{b^{*} x_{i}}\left[\begin{array}{ll}
x_{i} & \omega_{o} \\
a & b
\end{array}\right] \mathrm{F}_{x_{i}{ }^{*} b^{*}}\left[\begin{array}{ll}
b & \omega_{o} \\
a & x_{i}
\end{array}\right]=1 \\
& \mathrm{~F}_{1 k}\left[\begin{array}{cc}
i & j \\
i & j
\end{array}\right] \mathrm{F}_{k 1}\left[\begin{array}{cc}
i & i \\
j & j
\end{array}\right]=\frac{S_{1}{ }^{1} \cdot S_{1}{ }^{k}}{S_{1}{ }^{i} \cdot S_{1}{ }^{j}} \quad \mathrm{~F}_{1 x_{o}}\left[\begin{array}{cc}
x_{e} & \omega_{o} \\
x_{e} & \omega_{o}
\end{array}\right] \cdot \mathrm{F}_{x_{o} 1}\left[\begin{array}{cc}
\omega_{o} & \omega_{o} \\
x_{e} & x_{e}
\end{array}\right]=1 \\
& \mathrm{~F}_{k 1}\left[\begin{array}{cc}
i & i \\
j & j
\end{array}\right]=\frac{S_{1}{ }^{k}}{S_{1}{ }^{j}} \cdot \mathrm{F}_{j 1}\left[\begin{array}{cc}
k & k \\
i & i
\end{array}\right] \quad \mathrm{F}_{x_{o} 1}\left[\begin{array}{cc}
\omega_{o} & \omega_{o} \\
x_{e} & x_{e}
\end{array}\right]=\mathrm{F}_{x_{e} 1}\left[\begin{array}{cc}
\omega_{o} & \omega_{o} \\
x_{o} & x_{o}
\end{array}\right] \\
& \mathrm{F}_{11}\left[\begin{array}{cc}
i & i \\
i & i
\end{array}\right]=\frac{S_{1}{ }^{1}}{S_{1}{ }^{i}} \quad \mathrm{~F}_{11}\left[\begin{array}{cc}
\omega_{0} & \omega_{o} \\
\omega_{o} & \omega_{o}
\end{array}\right]=1 \\
& \mathrm{~F}_{p i}\left[\begin{array}{ll}
j & k \\
n & \ell
\end{array}\right] \mathrm{F}_{n 1}\left[\begin{array}{ll}
i & i \\
\ell & \ell
\end{array}\right]=\mathrm{F}_{n k}\left[\begin{array}{ll}
i & j \\
\ell & p
\end{array}\right] \mathrm{F}_{p 1}\left[\begin{array}{cc}
k & k \\
\ell & \ell
\end{array}\right] \\
& \sum_{s} e^{i \pi\left(h_{p}+h_{q}+2 h_{i}-2 h_{s}-\frac{1}{2}\left(h_{k}+h_{\ell}\right)\right)} \mathrm{F}_{k s}\left[\begin{array}{cc}
\bar{i} & p \\
i & q
\end{array}\right] \mathrm{F}_{s \ell}\left[\begin{array}{cc}
q & p \\
i & \bar{i}
\end{array}\right] \\
& =\sum_{m} e^{i \pi\left(-h_{p}-h_{q}-2 \bar{h}_{i}+2 h_{m}+\frac{1}{2}\left(h_{k}+h_{\ell}\right)\right)} \mathrm{F}_{k m}\left[\begin{array}{cc}
p & i \\
q & \bar{i}
\end{array}\right] \mathrm{F}_{m \ell}\left[\begin{array}{cc}
p & q \\
i & \bar{i}
\end{array}\right]
\end{aligned}
$$

\section{References}

[1] A.A. Belavin, A.M. Polyakov, A.B. Zamolodchikov, Infinite conformal symmetry in two-dimensional quantum field theory, Nucl. Phys. B241 (1984) 333-380.

[2] Ph. Di Francesco, P. Mathieu, D. Sénéchal, Conformal Field Theory, Springer 1998.

[3] Vl.S. Dotsenko, V.A. Fateev, Conformal algebra and multipoint correlation functions in 2d statistical models, Nucl. Phys. B240 (1984) 312-348. Four-point correlation functions and the operator agebra in 2d conformal invariant theories with central charge $c \leq 1$, Nucl. Phys. B251 (1985) 691-734. 
[4] A. Capelli, C. Itzykson, J.-B. Zuber, Modular invariant partition functions in two dimensions, Nucl. Phys. B280 (1987) 445-465.

[5] J.L. Cardy, Conformal invariance and surface critical behavior, Nucl. Phys. B240 (1984) $514-532$.

[6] J.L. Cardy, D.C. Lewellen, Bulk an boundary operators in conformal field theory, Phys. Lett. B259 (1991) 274-278.

[7] G. Pradisi, A. Sagnotti, Ya. S. Stanev, The open descendants of nondiagonal SU(2) WZW models Phys. Lett. B356 (1995) 230-238, hep-th/9506014. Completeness conditions for boundary operators in 2-D conformal field theory, Phys. Lett. B381 (1996) 97-104, hep-th/9603097.

[8] J. Fuchs, C. Schweigert, A Classifying algebra for boundary conditions, Phys. Lett. B414 (1997) 251-259, hep-th/9708141.

[9] R.E. Behrend, P.A. Pearce, J.-B. Zuber, Integrable boundaries, conformal boundary conditions and A-D-E fusion rules, J. Phys. A31 (1998) L763-L770, hep-th/9807142. R.E. Behrend, P.A. Pearce, V.B. Petkova, J.-B. Zuber, On the Classification of Bulk and Boundary Conformal Field Theories, Phys. Lett. B444 (1998) 163-166, hep-th/9809097.

[10] D.C. Lewellen, Sewing constraints for conformal field theories on surfaces with boundaries, Nucl. Phys. B372 (1992) 654-682.

[11] G. Moore and N. Seiberg, Lectures on RCFT, Physics, Geometry, and Topology, Plenum Press, New York, 1990.

[12] R.E. Behrend, P.A. Pearce, V.B. Petkova, J.-B. Zuber, Boundary Conditions in Rational Conformal Field Theories, Nucl. Phys. B570 (2000) 525-589, hep-th/9908036.

[13] J. Fuchs, A. Klemm, The computation of the operator algebra in nondiagonal conformal field theories, Annals Phys. 194 (1989) 303. V.B. Petkova, Structure constants of the (A,D) minimal $c<1$ conformal models, Phys. Lett. B225 (1989) 357-362.

[14] V.B. Petkova, J.-B. Zuber, On structure constants of sl(2) theories, Nucl. Phys. B438 (1995) 347-372, hep-th/9410209.

[15] I. Runkel, Boundary structure constants for the A-series Virasoro minimal models, Nucl. Phys. B549 (1999) 563-578, hep-th/9811178.

[16] W. Eholzer, M. Flohr, A. Honecker, R. Hubel, W. Nahm, R. Varnhagen, Representations of $W$-algebras with two generators and new rational models, Nucl. Phys. B383 (1992) 249-290. P. Bouwknegt, K. Schoutens, W-symmetry in conformal field theory, Phys. Rept. 223 (1993) 183-276, hep-th/9210010.

[17] L. Alvarez-Gaumé, C. Gomez, G. Sierra, Quantum group interpretation of some conformal field theories, Phys. Lett. B220 (1989) 142-152. G. Felder, J. Fröhlich, G. Keller, Braid matrices and structure constants for minimal conformal models, Comm. Math. Phys. 124 (1989) 647-664.

P. Furlan, A.Ch. Ganchev, V.B. Petkova, Fusion matrices and $c<1$ (quasi) local conformal field theories, Int. J. Mod. Phys. A5 (1990) 2721-2735.

[18] J.L. Cardy, Boundary conditions, fusion rules and the Verlinde formula, Nucl. Phys. B324 (1989) 581-596.

[19] P. Ruelle, Symmetric boundary conditions in boundary critical phenomena, J. Phys. A32 (1999) 8831-8850, hep-th/9904100.

[20] J.L. Cardy, I. Peschel, Finite size dependence of the free enery in two-dimensional critical systems, Nucl. Phys. B300 (1988) 377-399.

[21] V. Pasquier, Operator content of the ADE lattice models, J. Phys. A20 (1987) 5707-5733.

[22] J. Fuchs, C. Schweigert, Symmetry breaking boundaries. 1. General theory, Nucl. Phys. B558 (1999) 419-483, hep-th/9902132. Symmetry breaking boundaries. 2. More structures: Examples, Nucl. Phys. B568 (2000) 543-593, hep-th/9908025. 